\begin{tabular}{|c|l|}
\hline Title & Microscopic global optical potential for nucleon-nucleus systems in the energy range 50-400 MeV \\
\hline Author(s) & Furumoto, Takenori; Tsubakihara, Kohsuke; Ebata, S; Horiuchi, Wataru \\
\hline Citation & $\begin{array}{l}\text { Physical Review C, 99(3), 034605 } \\
\text { https://doi.org/10.1103/PhysRevC.99.034605 }\end{array}$ \\
\hline Issue Date & 2019-03-06 \\
\hline Doc URL & http://hdl.handle.net/2115/74949 \\
\hline Rights & @2019A A merican Physical Society \\
\hline Type & article \\
\hline File Information & PhysRevC.99.034605-1.pdf \\
\hline
\end{tabular}

Instructions for use 


\title{
Microscopic global optical potential for nucleon-nucleus systems in the energy range 50-400 MeV
}

\author{
T. Furumoto* \\ Graduate School of Education, Yokohama National University, Yokohama 240-8501, Japan \\ K. Tsubakihara \\ Laboratory for Advanced Nuclear Energy, Tokyo Institute of Technology, Tokyo 152-8550, Japan \\ S. Ebata \\ School of Environment and Society, Tokyo Institute of Technology, Tokyo 152-8550, Japan \\ and Reaction Nuclear Data Centre, Faculty of Science, Hokkaido University, Sapporo 060-0810, Japan \\ W. Horiuchi \\ Department of Physics, Hokkaido University, Sapporo 060-0810, Japan
}

(Received 2 September 2018; revised manuscript received 24 December 2018; published 6 March 2019)

\begin{abstract}
We provide a microscopic global optical potential (MGOP) for nucleon-nucleus $(N A)$ systems in a wide range of nuclear mass numbers $(A=10-276)$ and incident energies $(E=50-400 \mathrm{MeV})$. The potential is microscopically constructed based on a single-folding (SF) model with the complex $G$-matrix interaction. The nuclear densities used in the SF model are generated, in a nonempirical way, from two kinds of microscopic mean-field models: the relativistic-mean-field (RMF) and Skyrme-Hartree-Fock + BCS (HF+BCS) models. We calculate the $N A$ potentials for more than 1000 even-even nuclei with atomic number $Z=6-92$, involving proton- and neutron-rich unstable nuclei. We confirm that both the MGOP models well reproduce the available experimental data of the total reaction cross sections, the total neutron cross sections, the elastic-scattering cross sections, the analyzing power, and the spin-rotation function $Q$. We also calculate the proton scattering cross sections of ${ }^{22} \mathrm{O},{ }^{24} \mathrm{O}$, and ${ }^{56} \mathrm{Ni}$ targets to compare the experimental data and then the cross sections for unknown ${ }^{48} \mathrm{~S},{ }^{100} \mathrm{Zr}$, and ${ }^{110} \mathrm{Zr}$ are presented for future measurements. For the sake of convenience, the real and imaginary parts of the central and spin-orbit components of the $N A$ potentials are respectively represented in a linear combination of 12-range Gaussians. They are provided on the website [http://www2.yukawa.kyoto-u.ac.jp/ takenori.furumoto/] with a program source file for reconstructing the MGOP.
\end{abstract}

DOI: 10.1103/PhysRevC.99.034605

\section{INTRODUCTION}

The optical model potential (OMP) has often been used as a basic tool to analyze elastic-scattering phenomena as well as nonelastic ones [1]. Since the traditional OMP is derived through a phenomenological way with the help of the available experimental data, it is difficult to obtain the OMP for systems involving short-lived unstable nuclei, in which the number of experimental data is not sufficient. To obtain the OMP in such regions, the global optical potential (GOP) has been proposed. Historically, the GOP has been constructed phenomenologically, i.e., the parameters involved in the GOP are systematically determined so as to reproduce a number of the existing data. The OMP has been obtained even if there is no experimental data. The readers are referred to Refs. [2-10] for nucleon-nucleus (NA) systems, Refs. [11-15] for deuteron-nucleus systems, Refs. $[16,17]$ for ${ }^{3} \mathrm{He}$-nucleus systems, Refs. [18-20] for $\alpha$-nucleus systems, and Ref. [21] for ${ }^{6,7} \mathrm{Li}$-nucleus systems. The GOP has been successful in

\footnotetext{
*furumoto-takenori-py@ynu.ac.jp
}

describing a number of systems involving stable nuclei. The applicability of the GOP to the unstable nuclei has not yet been investigated well because the GOP has been applied to the unknown regions simply by the extrapolation of these parameters determined only with the stable nuclei. A reliable GOP applicable to exotic nuclei is desired as advancing of radioactive-ion beam facilities. The GOP models for unstable nuclei were constructed for the systems involving ${ }^{6} \mathrm{He}$ [22] and ${ }^{8} \mathrm{Li}$ [23]. However, their energy ranges were limited only up to $40 \mathrm{MeV} /$ nucleon due to the lack of the experimental data.

A global and microscopic description of the elastic scattering phenomena has been extended to systems involving unstable nuclei. Though the $N A$ elastic scattering has been intensively studied by many authors [24-40], the OMP parameters used in those studies have not always been given in a convenient form as a tool. Recently, the GOP for nucleusnucleus systems was constructed with the São Paulo global density [41] from a microscopic point of view [42]. Parameter sets of the OMP, i.e., the microscopic global optical potential (MGOP), for a wide range of mass numbers and incident energies can be obtained from the website [43]. 


\begin{tabular}{|c|c|c|c|c|c|c|c|c|c|c|c|c|c|c|c|c|c|c|}
\hline \multicolumn{19}{|c|}{ MGOP1 (with RMF model) } \\
\hline & 1 & 2 & 3 & 4 & 5 & 6 & 7 & 8 & 9 & 10 & 11 & 12 & 13 & 14 & 15 & 16 & 17 & 18 \\
\hline 1 & ${ }_{1} \mathrm{H}$ & & & & & & & & & & & & & & & & & ${ }_{2} \mathrm{He}$ \\
\hline 2 & $3 \mathrm{Li}$ & ${ }_{4} \mathrm{Be}$ & & & & & & & & & & & $5^{B}$ & $10-22{ }_{6} \mathrm{C}$ & ${ }_{7} \mathrm{~N}$ & $12-28{ }_{8} \mathrm{O}$ & ${ }_{9} \mathrm{~F}$ & $18-30_{10} \mathrm{Ne}$ \\
\hline 3 & ${ }_{11} \mathrm{Na}$ & $20-40_{12} \mathrm{Mg}$ & & & & & & & & & & & $13 \mathrm{Al}$ & $22-44_{14} \mathrm{Si}$ & ${ }_{15} \mathrm{P}$ & $26-48_{16} \mathrm{~S}$ & ${ }_{17} \mathrm{Cl}$ & $32-52_{18} \mathrm{Ar}$ \\
\hline 4 & $19 \mathrm{~K}$ & $34-56_{20} \mathrm{Ca}$ & $21 \mathrm{Sc}$ & $42-6222 \mathrm{Ti}$ & $23 \mathrm{~V}$ & $46-66_{24} \mathrm{Cr}$ & $25 \mathrm{Mn}$ & $48-72{ }_{26} \mathrm{Fe}$ & $27 \mathrm{Co}$ & $50-78_{28} \mathrm{Ni}$ & ${ }_{29} \mathrm{Cu}$ & $58-82_{30} \mathrm{Zn}$ & $31 \mathrm{Ga}$ & $58-88_{32} \mathrm{Ge}$ & 33 As & $66-94_{34} \mathrm{Se}$ & $35 \mathrm{Br}$ & $70-100_{36} \mathrm{Kr}$ \\
\hline 5 & $37 \mathrm{Rb}$ & $74-104_{38} \mathrm{Sr}$ & $39 \mathrm{Y}$ & $\frac{78-110_{40} \mathrm{Zr}}{}$ & ${ }_{41} \mathrm{Nb}$ & $86-11442 \mathrm{Mo}$ & $43 \mathrm{Tc}$ & $88-12044 \mathrm{Ru}$ & $45 \mathrm{Rh}$ & $92-124_{46} \mathrm{Pd}$ & $47 \mathrm{Ag}$ & $96-128_{48} \mathrm{Cd}$ & 49 In & $100-136_{50} \mathrm{Sn}$ & $51 \mathrm{Sb}$ & $108-142_{52} \mathrm{Te}$ & $53 \mathrm{I}$ & $110-146_{54} \mathrm{Xe}$ \\
\hline 6 & $55 \mathrm{Cs}$ & $114-152_{56} \mathrm{Ba}$ & & $156-188_{72} \mathrm{Hf}$ & $73 \mathrm{Ta}$ & $160-192{ }^{74} \mathrm{~W}$ & $75 \operatorname{Re}$ & $162-196_{76} \mathrm{Os}$ & $77 \mathrm{Ir}$ & $166-202_{78} \mathrm{Pt}$ & $79 \mathrm{Au}$ & $172-206{ }_{80} \mathrm{Hg}$ & $81 \mathrm{Ti}$ & $178-214{ }_{82} \mathrm{~Pb}$ & $83 \mathrm{Bi}$ & $188-220_{84} \mathrm{Po}$ & $85 \mathrm{At}$ & $196-228_{86} \mathrm{Rn}$ \\
\hline 7 & $87 \mathrm{Fr}$ & $202-234{ }_{88} \mathrm{Ra}$ & & $104 \mathrm{Rf}$ & $105 \mathrm{Db}$ & $106 \mathrm{Sg}$ & $107 \mathrm{Bh}$ & $108 \mathrm{Hs}$ & $109 \mathrm{Mt}$ & 110 Ds & $111 \mathrm{Rg}$ & $112 \mathrm{Cn}$ & $113^{\mathrm{Nh}}$ & $114 \mathrm{FI}$ & $115 \mathrm{Mc}$ & $116^{\mathrm{LV}}$ & $117 \mathrm{Ts}$ & $118 \mathrm{Og}$ \\
\hline \multicolumn{3}{|c|}{ Lanthanoid series } & $57 \mathrm{Ra}$ & $120-156_{58} \mathrm{Ce}$ & $59 \mathrm{Pr}$ & $124-160_{60} \mathrm{Nd}$ & $61 \mathrm{Pm}$ & $128-164_{62} \mathrm{Sm}$ & $63 \mathrm{Eu}$ & $134-168_{64} \mathrm{Gd}$ & $65 \mathrm{~Tb}$ & $142-172_{66} \mathrm{Dy}$ & $67 \mathrm{Ho}$ & $144-176_{68} \mathrm{Er}$ & $69 \mathrm{Tm}$ & $150-18070 \mathrm{Yb}$ & $71 \mathrm{Lu}$ & \\
\hline \multicolumn{3}{|c|}{ Actinoid series } & $89 \mathrm{Ac}$ & $210-238_{90} \mathrm{Th}$ & $91 \mathrm{~Pa}$ & $218-242_{92} \mathrm{U}$ & $93 \mathrm{~Np}$ & $94 \mathrm{Pu}$ & $95 \mathrm{Am}$ & $96 \mathrm{Cm}$ & $97 \mathrm{Bk}$ & $98 \mathrm{Cf}$ & $99 \mathrm{Es}$ & $100 \mathrm{Fm}$ & $101 \mathrm{Md}$ & 102 No & $103 \mathrm{Lr}$ & \\
\hline \multicolumn{19}{|c|}{ MGOP2 (with Skyrme HF+BCS model) } \\
\hline & 1 & 2 & 3 & 4 & 5 & 6 & 7 & 8 & 9 & 10 & 11 & 12 & 13 & 14 & 15 & 16 & 17 & 18 \\
\hline 1 & ${ }_{1} \mathrm{H}$ & & & & & & & & & & & & & & & & & $2 \mathrm{He}$ \\
\hline 2 & $3 \mathrm{Li}$ & ${ }_{4} \mathrm{Be}$ & & & & & & & & & & & ${ }_{5} \mathrm{~B}$ & $\underline{12-18}{ }_{6} \mathrm{C}$ & ${ }_{7} \mathrm{~N}$ & $\underline{16-24}{ }_{8} \mathrm{O}$ & ${ }_{9} \mathrm{~F}$ & $20-30{ }_{10} \mathrm{Ne}$ \\
\hline & $11 \mathrm{Na}$ & $24-36_{12} \mathrm{Mg}$ & & & & & & & & & & & ${ }_{13} \mathrm{Al}$ & $\underline{28-42_{14} \mathrm{Si}}$ & ${ }_{15} \mathrm{P}$ & $\underline{32-48}{ }_{16} \mathrm{~S}$ & $17 \mathrm{Cl}$ & $36-54{ }_{18} \mathrm{Ar}$ \\
\hline 4 & $19 \mathrm{~K}$ & $40-60_{20} \mathrm{Ca}$ & $21 \mathrm{Sc}$ & $\underline{44-66} 22 \mathrm{Ti}$ & $23 \mathrm{~V}$ & $48-7224 \mathrm{Cr}$ & $25 \mathrm{Mn}$ & $52-7826 \mathrm{Fe}$ & $27 \mathrm{Co}$ & $\underline{56-84}{ }_{28} \mathrm{Ni}$ & ${ }_{29} \mathrm{Cu}$ & $60-90_{30} \mathrm{Zn}$ & $31 \mathrm{Ga}$ & $64-96_{32} \mathrm{Ge}$ & ${ }_{33} \mathrm{As}$ & $68-102_{34 \mathrm{Se}}$ & $35 \mathrm{Br}$ & $72-108_{36} \mathrm{Kr}$ \\
\hline & $37 \mathrm{Rb}$ & $76-114_{38} \mathrm{Sr}$ & $39 \mathrm{Y}$ & $80-120_{40} \mathrm{Zr}$ & ${ }_{41} \mathrm{Nb}$ & $84-126_{42} \mathrm{Mo}$ & $43 \mathrm{Tc}$ & $88-13244 \mathrm{Ru}$ & $45 \mathrm{Rh}$ & $92-138_{46} \mathrm{Pd}$ & $47 \mathrm{Ag}$ & $96-144_{48} \mathrm{Cd}$ & 49In & $100-150_{50} \mathrm{Sn}$ & $51 \mathrm{Sb}$ & $110-156_{52} \mathrm{Te}$ & $53 \mathrm{I}$ & $110-162_{54} \mathrm{Xe}$ \\
\hline 6 & $55 \mathrm{Cs}$ & $116-168_{56} \mathrm{Ba}$ & & $162-216_{72} \mathrm{Hf}$ & $73 \mathrm{Ta}$ & $166-22274 \mathrm{~W}$ & $75 \operatorname{Re}$ & $170-22876 \mathrm{Os}$ & $77 \mathrm{Ir}$ & $176-23478 \mathrm{Pt}$ & $79 \mathrm{Au}$ & $176-240{ }_{80} \mathrm{Hg}$ & $81^{\mathrm{Ti}}$ & $182-246{ }_{82} \mathrm{~Pb}$ & $83 \mathrm{Bi}$ & $206-252{ }_{84} \mathrm{Po}$ & $85 A t$ & $210-258{ }_{86} \mathrm{Rn}$ \\
\hline 7 & $87 \mathrm{Fr}$ & $214-258_{88} \mathrm{Ra}$ & & $104 \mathrm{Rf}$ & $105 \mathrm{Db}$ & $106 \mathrm{Sg}$ & $107 \mathrm{Bh}$ & $108 \mathrm{Hs}$ & $109 \mathrm{Mt}$ & 110 Ds & $111 \mathrm{Rg}$ & $112 \mathrm{Cn}$ & $113 \mathrm{Nh}$ & $114 \mathrm{FI}$ & $115 \mathrm{Mc}$ & $116 \mathrm{LV}$ & $117 \mathrm{Ts}$ & $118 \mathrm{Og}$ \\
\hline \multicolumn{3}{|c|}{ Lanthanoid series } & $57 \mathrm{Ra}$ & $120-174_{58} \mathrm{Ce}$ & $59 \mathrm{Pr}$ & $126-180_{60} \mathrm{Nd}$ & $6{ }_{61} \mathrm{Pm}$ & $130-186_{62} \mathrm{Sm}$ & $63 \mathrm{Eu}$ & $140-192_{64} \mathrm{Gd}$ & $65 \mathrm{~Tb}$ & $144-198_{66} \mathrm{Dy}$ & $67 \mathrm{Ho}$ & $148-204_{68} \mathrm{Er}$ & $69 \mathrm{Tm}$ & $150-210_{70} \mathrm{Yb}$ & $71 \mathrm{Lu}$ & \\
\hline \multicolumn{3}{|c|}{ Actinoid series } & $89 \mathrm{Ac}$ & $214-270_{90} \mathrm{Th}$ & $91 \mathrm{~Pa}$ & $216-276_{92} \mathrm{U}$ & $93 \mathrm{~Np}$ & $94 \mathrm{Pu}$ & $95 \mathrm{Am}$ & ${ }_{96} \mathrm{Cm}$ & $97 \mathrm{Bk}$ & $98 \mathrm{Cf}$ & $99 \mathrm{Es}$ & ${ }_{100} \mathrm{Fm}$ & $101 \mathrm{Md}$ & $102 \mathrm{No}$ & $103 \mathrm{Lr}$ & \\
\hline
\end{tabular}

FIG. 1. Display examples of tables shown in the website [43].

However, the reliability of this MGOP model is questionable for the systems near the dripline as discussed in Ref. [42] because the input global density is not optimized for unstable nuclei.

In this paper, we provide a new MGOP for the $N A$ systems. The MGOP is calculated by a single-folding (SF) model with the complex $G$-matrix interaction [44]. Input densities needed in the SF model are obtained in a nonempirical way by microscopic mean-field models: the relativistic-mean-field (RMF) [45,46] and Hartree-Fock + BCS (HF + BCS) [47] models, for almost the whole mass regions. Although they are limited to even-even nuclei, the total number of nuclides treated in this paper is about 1000 . We calculate the total reaction cross sections, the elastic-scattering cross sections, and the analyzing power, and compare them to the available experimental data.

In the next section, we first explain our theoretical models. The SF model calculation and the derivation of the nuclear densities by the RMF and HF + BCS models are briefly described, and then the density profiles obtained with these models are compared. The MGOP is provided as a functional form of a sum of 12-range Gaussians, which is defined in Sec. III. These parameters for a number of nuclei in a wide range of mass numbers and incident energies can be obtained from the website [43] as illustrated in Fig. 1. In Sec. IV, the two MGOP models are tested in comparison with the existing experimental data of the elastic-scattering cross sections and analyzing power of the $N A$ scattering. A summary is given in Sec. V.

\section{THEORETICAL MODELS}

Throughout this paper, we use the normal kinematics in which the incident nucleon is scattered by the target nucleus though the inverse kinematics is used for unstable nuclei. The OMP for the $N A$ system is written as

$$
U=V_{\mathrm{CE}}+i W_{\mathrm{CE}}+\left(V_{\mathrm{SO}}+i W_{\mathrm{SO}}\right) \ell \cdot \boldsymbol{\sigma}+V_{\mathrm{Coul}},
$$

where $V_{\mathrm{CE}}$ and $V_{\mathrm{SO}}\left(W_{\mathrm{CE}}\right.$ and $\left.W_{\mathrm{SO}}\right)$ denote a real (imaginary) parts of the central and spin-orbit components of the OMP, respectively, and $V_{\text {Coul }}$ is the Coulomb potential term that only appears in the proton-nucleus $(p A)$ system.

In Sec. II A, we explain the SF model to construct the OMP for the NA systems. Theoretical inputs to the SF model are the nuclear densities for the proton and neutron. In Sec. II B, the derivation of these input densities with the RMF and $\mathrm{HF}+\mathrm{BCS}$ models is explained. A comparison of the resulting density profiles is made by calculating the charge radii and the root-mean-square (rms) radii of the point-neutron, pointproton, and point-matter densities with these structure models.

\section{A. Single-folding model}

The central and spin-orbit components of the OMP in Eq. (1) are obtained as complex numbers by the SF procedure with the input nuclear densities and the complex $G$-matrix interaction. Therefore, both the real and imaginary parts of the OMP can be obtained in a nonempirical way. We employ the MPa $G$-matrix interaction $[48,49]$ which is derived from 
the realistic nucleon-nucleon interaction, ESC08 [50]. The $\mathrm{MPa}$ interaction takes into account the three-body force effects with the multipomeron exchange potential [50]. The three-body force induces additional repulsion to the OMP and is known to have an important role to improve the analyzing power at forward angles at incident energy from 100 to 200
$\mathrm{MeV}$ [36]. The MPa interaction has been widely applied not only in the nucleus-nucleus scattering [51-54], but also hypernucler systems [55,56] and neutron matter [48,49,57].

For the central component, we calculate the SF potential in the same manner as described in Refs. [31,36]. The SF potential at a given incident energy $E$ can be written by

$$
\begin{aligned}
V_{\mathrm{CE}}(R ; E)+i W_{\mathrm{CE}}(R ; E)= & \int\left\{\rho_{p}(\boldsymbol{r}) v_{N p}^{\mathrm{DI}}\left(\boldsymbol{s}, k_{F}^{(N p)} ; E\right)+\rho_{n}(\boldsymbol{r}) v_{N n}^{\mathrm{DI}}\left(\boldsymbol{s}, k_{F}^{(N n)} ; E\right)\right\} d \boldsymbol{r} \\
& +\int\left\{\tilde{\rho}_{p}\left(\boldsymbol{R}, \boldsymbol{r}^{\prime}\right) v_{N p}^{\mathrm{EX}}\left(\boldsymbol{s}, k_{F}^{(N p)} ; E\right)+\tilde{\rho}_{n}\left(\boldsymbol{R}, \boldsymbol{r}^{\prime}\right) v_{N n}^{\mathrm{EX}}\left(\boldsymbol{s}, k_{F}^{(N n)} ; E\right)\right\} j_{0}(k s) d \boldsymbol{r}^{\prime},
\end{aligned}
$$

where $\rho_{p}$ and $\rho_{n}$ are the proton and neutron densities of the target nucleus, respectively. The coordinate $\boldsymbol{s}=\boldsymbol{r}-\boldsymbol{R}$ denotes the relative vector between the incident nucleon and a nucleon in the target nucleus. The direct $(x=\mathrm{DI})$ and exchange ( $x=\mathrm{EX})$ parts of the $G$-matrix interaction for the nucleonproton $(N p)$ and nucleon-neutron $(N n)$ are denoted as $v_{N p}^{x}$ and $v_{N n}^{x}$, respectively. The local momentum $k$ of the $p A$ system which appears in the second term of Eq. (2) is defined by

$$
k^{2}=\frac{2 \mu}{\hbar^{2}}\left(E_{\mathrm{c} . \mathrm{m} .}-V_{\mathrm{CE}}(R ; E)-V_{\mathrm{Coul}}(R)\right),
$$

where $E_{\text {c.m. }}$ and $\mu$ are the center-of-mass energy and the reduced mass of the $p A$ system, respectively. A uniform charge distribution is assumed for the Coulomb potential, $V_{\text {Coul }}$, with a radius of $R_{C}=1.25 A_{T}^{1 / 3} \mathrm{fm}$, where $A_{T}$ is the nucleon number of the target nucleus. For the neutron-nucleus $(n A)$ system, $k$ is obtained simply by omitting the Coulomb term.

The Fermi momenta for the proton and neutron $k_{F}^{(p)}=$ $\left(3 \pi^{2} \rho_{p}\right)^{1 / 3}$ and $k_{F}^{(n)}=\left(3 \pi^{2} \rho_{n}\right)^{1 / 3}$ are, in general, different in a finite nucleus. We evaluate the density dependence of the complex $G$-matrix interaction with the Fermi momenta taking differently for the $p p$ and $n n$ interactions in Eq. (2) following the prescription given in Ref. [32]

$$
\begin{aligned}
k_{F}^{(p p)} & =k_{F}^{(p)}, \\
k_{F}^{(n n)} & =k_{F}^{(n)}, \\
k_{F}^{(p n)} & =k_{F}^{(n p)}=\frac{1}{2}\left(k_{F}^{(p)}+k_{F}^{(n)}\right) .
\end{aligned}
$$

Here the local densities $\rho_{p}$ and $\rho_{n}$ are evaluated at the position of the nucleon in the target nucleus, $r$. The density matrix $\tilde{\rho}\left(\boldsymbol{r}, \boldsymbol{r}^{\prime}\right)$ is approximated in the same manner as in Ref. [58]

$$
\begin{aligned}
& \tilde{\rho}_{p}\left(\boldsymbol{r}, \boldsymbol{r}^{\prime}\right)=\frac{3}{k_{F, N p}^{\mathrm{eff}} s} j_{1}\left(k_{F, N p}^{\mathrm{eff}} s\right) \rho_{p}\left(\frac{\boldsymbol{r}+\boldsymbol{r}^{\prime}}{2}\right), \\
& \tilde{\rho}_{n}\left(\boldsymbol{r}, \boldsymbol{r}^{\prime}\right)=\frac{3}{k_{F, N n}^{\mathrm{eff}} s} j_{1}\left(k_{F, N n^{\mathrm{eff}}}^{\mathrm{eff}}\right) \rho_{n}\left(\frac{\boldsymbol{r}+\boldsymbol{r}^{\prime}}{2}\right),
\end{aligned}
$$

where $k_{F, N p}^{\mathrm{eff}}$ and $k_{F, N n}^{\mathrm{eff}}$ are the effective Fermi momenta that appear in the density matrix expansion $[58,59]$

$$
\begin{aligned}
& k_{F, p p}^{\mathrm{eff}}=\left[\left(3 \pi^{2} \rho_{p}\right)^{2 / 3}+\frac{5 C_{\mathrm{s}}\left(\nabla \rho_{p}\right)^{2}}{3 \rho_{p}^{2}}+\frac{5 \nabla^{2} \rho_{p}}{36 \rho_{p}}\right]^{1 / 2}, \\
& k_{F, n n}^{\mathrm{eff}}=\left[\left(3 \pi^{2} \rho_{n}\right)^{2 / 3}+\frac{5 C_{\mathrm{s}}\left(\nabla \rho_{n}\right)^{2}}{3 \rho_{n}^{2}}+\frac{5 \nabla^{2} \rho_{n}}{36 \rho_{n}}\right]^{1 / 2}, \\
& k_{F, p n}^{\mathrm{eff}}=k_{F, n p}^{\mathrm{eff}}=\frac{1}{2}\left(k_{F, p p}^{\mathrm{eff}}+k_{F, n n}^{\mathrm{eff}}\right),
\end{aligned}
$$

where we adopt $C_{\mathrm{s}}=1 / 4$ [60].

The spin-orbit potential is calculated by folding the spinorbit part of the complex $G$-matrix interaction with the nucleon density of the target nucleus, which is also composed of the direct and exchange parts as

$$
U_{\mathrm{SO}}(\boldsymbol{R} ; E)=U_{\mathrm{SO}}^{\mathrm{DI}}(R ; E)+U_{\mathrm{SO}}^{\mathrm{EX}}(R ; E)
$$

with

$$
\begin{aligned}
U_{\mathrm{SO}}^{\mathrm{DI}}(R ; E) & =\frac{1}{4 R^{2}} \int \boldsymbol{R} \cdot(\boldsymbol{R}-\boldsymbol{r})\left\{\rho_{p}(\boldsymbol{r}) v_{\mathrm{SO}, N p}^{\mathrm{DI}}\left(\boldsymbol{s}, k_{F}^{(N p)} ; E\right)+\rho_{n}(\boldsymbol{r}) v_{\mathrm{SO}, N n}^{\mathrm{DI}}\left(\boldsymbol{s}, k_{F}^{(N n)} ; E\right)\right\} d \boldsymbol{r}, \\
U_{\mathrm{SO}}^{\mathrm{EX}}(R ; E) & =\sum_{\alpha=n, p} \pi \int d s s^{3}\left[\frac{2 j_{0}(k s)}{R} \rho_{1}^{(N \alpha)}(R, s)+\frac{j_{1}(k s)}{2 k} \delta_{0}^{(N \alpha)}(R, s)\right],
\end{aligned}
$$

where $\alpha$ runs $p$ and $n$ for the target nucleus, and

$$
\begin{aligned}
\delta_{0}^{(N \alpha)}(R, s)= & \frac{1}{2} \int_{-1}^{+1} d q \frac{v_{\mathrm{SO}, N \alpha}^{\mathrm{EX}}\left(s, k_{F}^{(N \alpha)} ; E\right)}{\sqrt{R^{2}+s^{2} / 4+R s q}}\left[\left.\frac{3}{k_{F, N \alpha}^{\mathrm{eff}} s} j_{1}\left(k_{F, N \alpha}^{\mathrm{eff}} s\right) \frac{d}{d x} \rho_{\alpha}(x)\right|_{x=\sqrt{R^{2}+s^{2} / 4+R s q}}\right. \\
& \left.+\left.\left.s \rho_{\alpha}\left(\sqrt{R^{2}+s^{2} / 4+R s q}\right) \frac{d}{d x} k_{F, N \alpha}^{\mathrm{eff}}(x)\right|_{x=\sqrt{R^{2}+s^{2} / 4+R s q}} \frac{d}{d y}\left(\frac{3}{y} j_{1}(y)\right)\right|_{y=k_{F, N \alpha}^{\mathrm{eff}} s}\right],
\end{aligned}
$$




$$
\rho_{1}^{(N \alpha)}(R, s)=\frac{1}{2} \int_{-1}^{+1} d q q v_{\mathrm{SO}, N \alpha}^{\mathrm{EX}}\left(s, k_{F}^{(N \alpha)} ; E\right) \frac{3}{k_{F, N \alpha}^{\mathrm{eff}} s} j_{1}\left(k_{F, N \alpha}^{\mathrm{eff}} s\right) \rho_{\alpha}\left(\sqrt{R^{2}+s^{2} / 4+R s q}\right) .
$$

The above equations are calculated in the same way as in Ref. [36].

\section{B. Nucleon density with microscopic mean-field models}

As shown in the previous subsection, the proton and neutron densities $\left(\rho_{p}\right.$ and $\left.\rho_{n}\right)$ are required in the SF model calculation as inputs. In the present MGOP, fully self-consistent microscopic structure models are employed to extend the GOP toward the NA systems including unknown proton- and neutron-rich unstable nuclei. To see the structure model dependence, we employ (i) RMF [45,46] and (ii) HF + BCS [47] models. We briefly explain how the densities are generated from these structure models.

\section{1. $R M F$ model}

The RMF model Lagrangian consists of the nucleon Lagrangian $\mathcal{L}_{\mathrm{N}}$ with meson-baryon couplings, and the meson Lagrangian $\mathcal{L}_{\mathrm{M}}$ with the effective meson potential $V_{M}$. If we adopt the time-reversal (static) ansatz to the RMF Lagrangian, $\mathcal{L}_{\mathrm{N}}$ reads

$$
\mathcal{L}_{\mathrm{N}}=\bar{\psi}_{N}\left[i \not \partial-M_{N}^{*}\left(\varphi_{\sigma}\right)-\gamma^{0} U_{v}\left(\omega, R_{0}, A_{0}\right)\right] \psi_{N}
$$

with

$$
\begin{gathered}
M_{N}^{*}\left(\varphi_{\sigma}\right)=M_{N}-g_{\sigma} \varphi_{\sigma}, \\
U_{v}\left(\omega, R_{0}, A_{0}\right)=g_{\omega} \omega+g_{\rho} \tau_{3} R_{0}+e \frac{1+\tau_{3}}{2} A_{0},
\end{gathered}
$$

where $\varphi_{\sigma}, \omega, R_{0}$ correspond to the scalar-isoscalar, vectorisoscalar, and vector-isovector meson fields, respectively. $g_{\sigma}$, $g_{\omega}$, and $g_{\rho}$ denote the coupling between these mesons and nucleon, respectively. The effect of the Coulomb interaction is taken into account by the photon field $A_{0}$. The effective mass of the nucleon $M_{N}^{*}\left(\varphi_{\sigma}\right)$ is, in general, smaller than that of the nucleon mass in the vacuum because the coupling between the scalar meson and the nucleon is attractive. $\tau_{3}$ is a projection operator of $z$-component of the isospin and that yields $\tau_{3} \chi_{s}(t)= \pm \chi_{s}(t)$, where $\chi_{s}(t)$ is the isospin wave function of nucleon and $t=+1 / 2(p)$ or $-1 / 2(n)$. In this paper, the Chiral SU(3) potential derived from the analytic SCL-LQCD (SCL3) is applied to $\mathcal{L}_{\mathrm{M}}$ as $V_{M}[45,46]$, and is written as

$$
U=\frac{1}{2} m_{\sigma}^{2} \varphi_{\sigma}^{2}+\frac{1}{2} m_{\zeta}^{2} \varphi_{\zeta}^{2}+V_{M}\left(\varphi_{\sigma}, \varphi_{\zeta}\right)
$$

with

$$
V_{M}=a_{\mathrm{SCL}}\left\{2 f_{\mathrm{SCL}}\left(\frac{\varphi_{\sigma}}{f_{\pi}}\right)+f_{\mathrm{SCL}}\left(\frac{\varphi_{\zeta}}{f_{\zeta}^{\prime}}\right)\right\}+\xi_{\sigma \zeta} \varphi_{\sigma} \varphi_{\zeta},
$$

and

$$
\begin{aligned}
a_{\mathrm{SCL}} & =\frac{1}{4}\left(m_{\sigma}^{2}+m_{\pi}^{2}\right), \\
f_{\mathrm{SCL}}(t) & =\log (1-t)-t-\frac{t^{2}}{2},
\end{aligned}
$$

where $f_{\pi}$ and $f_{\zeta}=f_{\zeta}^{\prime}-m_{s}$ are the expectation values of the scalar meson condensate. Because of the coupling between the $\sigma$ and $\zeta$ mesons consisting of the $s \bar{s}$ condensate in Eq. (21), the expectation value of the $\zeta$ scalar-isoscalar meson is finite even though it does not couple to the "normal" nucleon. Including $V_{M}$ shown in Eq. (21), $\mathcal{L}_{\mathrm{M}}$ is defined as follows:

$$
\begin{aligned}
\mathcal{L}_{\mathrm{M}}= & -\frac{1}{2}\left(\nabla \varphi_{\sigma}\right)^{2}-\frac{1}{2} m_{\sigma}^{2} \varphi_{\sigma}^{2}-V_{M}\left(\varphi_{\sigma}, \varphi_{\zeta}\right)-\frac{1}{2}\left(\nabla \varphi_{\zeta}\right)^{2} \\
& -\frac{1}{2} m_{\zeta}^{2} \varphi_{\zeta}^{2}+\frac{1}{2}(\nabla \omega)^{2}+\frac{1}{2} m_{\omega}^{2} \omega^{2}+\frac{c_{\omega}}{4} \omega^{4} \\
& +\frac{1}{2}\left(\nabla R_{0}\right)^{2}+\frac{1}{2} m_{\rho}^{2} R_{0}^{2}+\frac{1}{2}\left(\nabla A_{0}\right)^{2} .
\end{aligned}
$$

In Eq. (24), we additionally introduce a self-interaction potential of the $\omega$ meson in a quadratic form of the $\omega$ field and it is controlled by the parameter $c_{\omega}$. The SCL3 parameter set [46] is applied to the coupling constants, masses, $\xi_{\sigma \zeta}$, and $c_{\omega}$ in the present RMF model. The set is determined so as to reproduce the experimental $B / A$ values for finite nuclei, the charge radii of the typical stable nuclei, and the empirical saturation property of the nuclear matter.

The Dirac equations of each single-particle state $\psi_{i}$ are derived from the RMF Lagrangian. A single-particle energy is introduced as the Lagrange multiplier and Euler-Lagrange equations of nucleon and mesons derived from varying the RMF Lagrangian $\mathcal{L}=\mathcal{L}_{\mathrm{N}}+\mathcal{L}_{\mathrm{M}}$ defined in Eqs. (17) and (24). For simplicity, we adopt the spherical single-particle wave function

$$
\psi_{i}=\left(\begin{array}{c}
F_{i}(r) \Phi_{l_{i} j_{i} m_{i}}(\hat{\boldsymbol{r}}) \\
i G_{i}(r) \Phi_{\tilde{l}_{i} j_{i} m_{i}}(\hat{\boldsymbol{r}})
\end{array}\right) \chi_{s}(t),
$$

where $F_{i}$ and $G_{i}$ are the so-called large and small components coming from the relativistic treatment, respectively. Though the calculation is limited only to spherical cases, the spin-orbit splitting of the single-particle level is naturally taken into account through the difference between the large and small components $\left(l_{i}=j_{i} \mp 1 / 2, \tilde{l}_{i}=j_{i} \pm 1 / 2\right)$.

The scalar and vector densities are calculated with the radial part of the RMF single-particle wave functions

$$
\begin{aligned}
& \rho_{V}^{(n \text { or } p)}=\sum_{i \in p \text { or } n} \bar{\psi}_{i} \gamma^{0} \psi_{i} \sum_{i \in p \text { or } n}\left(\left|F_{i}\right|^{2}+\left|G_{i}\right|^{2}\right), \\
& \rho_{S}^{(n \text { or } p)}=\sum_{i \in p \text { or } n} \bar{\psi}_{i} \psi_{i} \sum_{i \in p \text { or } n}\left(\left|F_{i}\right|^{2}-\left|G_{i}\right|^{2}\right),
\end{aligned}
$$

where the summation runs over the number of proton or neutron, respectively. Both $\rho_{V}$ and $\rho_{S}$ are needed to solve Euler-Lagrange equations derived from RMF Lagrangian self-consistently because the vector and scalar meson fields depend on each densities, respectively. To obtain the input densities to the SF model calculation, we perform the centerof-mass correction to the vector density $\rho_{V}^{(p, n)}$ with the prescription given in Ref. [61]. 


\section{2. $\mathrm{HF}+\mathrm{BCS}$ model}

We explain how we obtain the ground-state densities with the nonrelativistic HF + BCS model. A fully self-consistent calculation is performed with the Skyrme effective interaction. Each single-particle state in a mean-field potential is solved in the three-dimensional coordinate space without any assumption of the nuclear shape.

The wave function of the HF + BCS model is obtained by solving the $\mathrm{HF}$ and BCS equations [62]

$$
\begin{aligned}
{[h, \rho] } & =0, \\
2 \tilde{\varepsilon}_{k} u_{k} v_{k}+\Delta_{k}\left(v_{k}^{2}-u_{k}^{2}\right) & =0, \quad k>0,
\end{aligned}
$$

where $h$ and $\rho$ are the single-particle Hamiltonian and density matrix, respectively. $v_{k}$ is the so-called occupation probability with $v_{k}^{2}+u_{k}^{2}=1$, and

$$
\tilde{\varepsilon}_{k}=\frac{1}{2}\left(\varepsilon_{k}+\varepsilon_{\bar{k}}\right)-\lambda,
$$

where $\varepsilon_{k}\left(=\left\langle\psi_{k}|\hat{h}| \psi_{k}\right\rangle\right)$ and $\lambda$ are the single-particle energy of $k$ state and the chemical potential, respectively. $\bar{k}$ denotes the conjugate of $k$. The constant-monopole-type pairing with a smooth truncation is employed for the pairing potential

$$
\Delta_{k}=\sum_{l} G_{k l} u_{l} v_{l}
$$

with $G_{k l}=g f\left(\varepsilon_{k}\right) f\left(\varepsilon_{l}\right)$, where $f(\varepsilon)$ is the cutoff function $[47,63]$. The amplitude $g$ is determined in the same manner as in Ref. [63].

We use the three-dimensional (3D) Cartesian coordinatespace representation for the canonical states $\psi_{k}(\mathrm{r}, \sigma)=$ $\left\langle\mathrm{r}, \sigma \mid \psi_{k}\right\rangle$ with $\sigma= \pm 1 / 2$. The intrinsic density in the bodyfixed frame can be obtained as

$$
\rho(\boldsymbol{r})=\sum_{k, \sigma} v_{k}^{2}\left|\psi_{k}(\boldsymbol{r}, \sigma)\right|^{2} .
$$

The HF + BCS densities in the laboratory frame are obtained from the angle-averaged intrinsic density [64]

$$
\rho_{\alpha}(r)=\frac{1}{4 \pi} \int d \hat{\boldsymbol{r}} \rho_{\alpha}(\boldsymbol{r})
$$

for proton $(\alpha=p)$ and neutron $(\alpha=n)$. Also the center-ofmass correction for the $\mathrm{HF}+\mathrm{BCS}$ densities is made as was done for the RMF densities in the present paper. Here we employ the $\mathrm{SkM}^{*}$ parameter set [65] as the effective two-body interaction which is known to describe well the properties of deformed nuclei. One can see the validity of those obtained densities in systematic analyses of the total reaction cross sections $[64,66]$ in which the nuclear deformation plays a decisive role to reproduce the cross section data. The analyses were further extended to various mass regions with useful applications [67-69].

\section{Comparison between RMF and HF + BCS models}

We perform the RMF calculations for the even-even nuclei in the range of $A=10-242$, and exclude some states having a positive single-particle energy, which are unphysical. The $\mathrm{HF}+\mathrm{BCS}$ densities are calculated in the range of the neutron number from $Z$ to $2 Z$ excluding unphysical results which

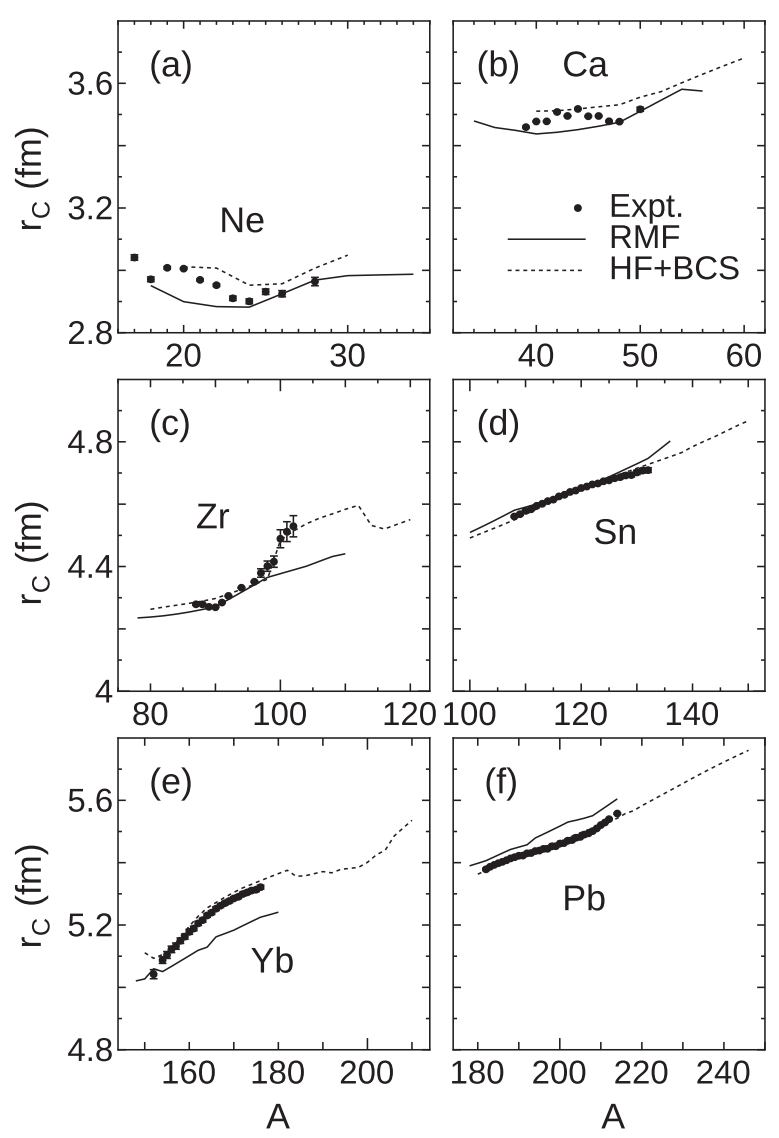

FIG. 2. Comparison of charge radii $r_{C}$ calculated from the RMF and $\mathrm{HF}+\mathrm{BCS}$ densities of (a) $\mathrm{Ne}$, (b) $\mathrm{Ca}$, (c) $\mathrm{Zr}$, (d) Sn, (e) $\mathrm{Yb}$, and (f) $\mathrm{Pb}$ isotopes. The experimental data are taken from Ref. [70].

give the separation energy (chemical potential) smaller than $2 \mathrm{MeV}$. As a result, the densities obtained with the RMF and $\mathrm{HF}+\mathrm{BCS}$ models are given in the different mass ranges as displayed in Fig. 1.

First, we compare the charge radii obtained by the RMF and $\mathrm{HF}+\mathrm{BCS}$ models. The charge densities are calculated by folding the point-proton densities obtained from the RMF and $\mathrm{HF}+\mathrm{BCS}$ models with the proton-charge form factor [71]. Figure 2 plots the charge radii for $\mathrm{Ne}, \mathrm{Ca}, \mathrm{Zr}, \mathrm{Sn}$, $\mathrm{Yb}$, and $\mathrm{Pb}$ isotopes obtained by the RMF (solid line) and $\mathrm{HF}+\mathrm{BCS}$ (dotted line) models. The experimental data are also plotted for comparison. We see the $\mathrm{HF}+\mathrm{BCS}$ model better reproduces the experimental data, whereas the RMF model shows some discrepancies, especially in the neutronrich regions. The deviation of the RMF results from the experimental data becomes large at most about $5 \%$ for $\mathrm{Ne}, \mathrm{Zr}$, and $\mathrm{Yb}$ isotopes, where the nuclear deformation plays a decisive role to change their density profiles, whereas the spherical mean field is assumed for the RMF calculations in the present paper.

Next we compare the density distributions obtained by the $\mathrm{RMF}$ and $\mathrm{HF}+\mathrm{BCS}$ models. We show the results of $\mathrm{S}$ and $\mathrm{Zr}$ isotopes in which the density profiles obtained from the RMF and $\mathrm{HF}+\mathrm{BCS}$ models are quite different. Figures 3 and 4 plot the point-neutron, proton, and matter density distributions 


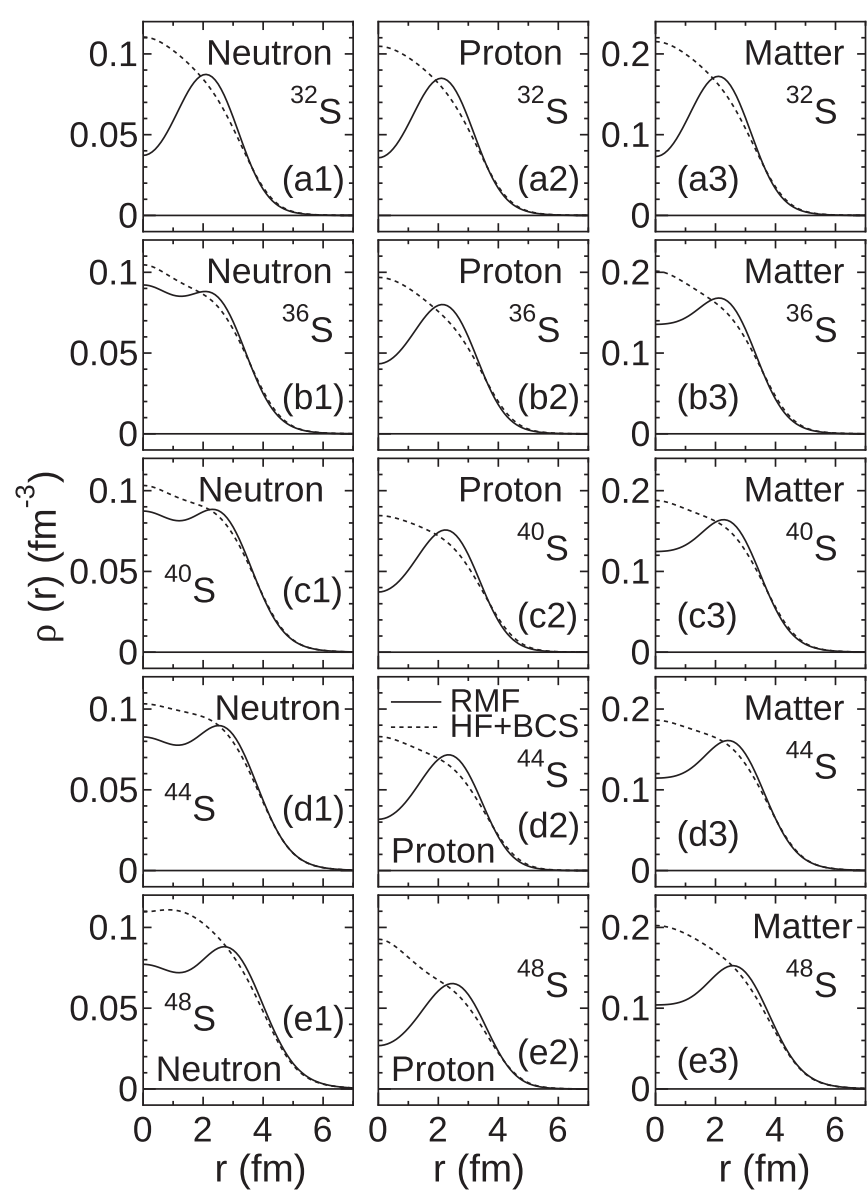

FIG. 3. Point-neutron, proton, and matter density distributions of (a) ${ }^{32} \mathrm{~S}$, (b) ${ }^{36} \mathrm{~S}$, (c) ${ }^{40} \mathrm{~S}$, (d) ${ }^{44} \mathrm{~S}$, and (e) ${ }^{48} \mathrm{~S}$ obtained with the RMF and $\mathrm{HF}+\mathrm{BCS}$ models.

of the selected $\mathrm{S}$ and $\mathrm{Zr}$ isotopes. For the $\mathrm{S}$ isotopes, the difference of the densities obtained from the RMF and HF + BCS models is clearly seen in the surface region. In the inner region of the $S$ isotopes, the so-called bubble structure $[72,73]$ appears in the RMF calculations.

For $\mathrm{Zr}$ isotopes, the RMF and HF + BCS models also show some difference in the surface and inner regions. The $\mathrm{HF}+\mathrm{BCS}$ densities show almost constant behavior in the internal regions, while the RMF densities exhibit some oscillatory behavior. For the spherical RMF densities, we see the characteristics of the single-particle states. The deformation of the $\mathrm{Zr}$ isotopes strongly depend on the number of the excess neutrons. This characteristic may disappear through the angle-averaged procedure in Eq. (33).

We note that the charge radii of the $\mathrm{Zr}$ isotopes obtained by the present models show the large difference as displayed in Fig. 2, whereas the rms radii of the point-matter densities are similar to each other: The RMF (HF + BCS) model gives 4.111, 4.251, 4.560, and $4.665 \mathrm{fm}(4.137,4.244,4.516$, and $4.698 \mathrm{fm}$ ) for rms radii of the point-matter densities of the ${ }^{80} \mathrm{Zr}$, ${ }^{90} \mathrm{Zr}$, ${ }^{100} \mathrm{Zr}$, and ${ }^{110} \mathrm{Zr}$ nuclei, respectively. Here we compared our results for the selected nuclei. We also list, in the website [43], the values of the charge radii and the

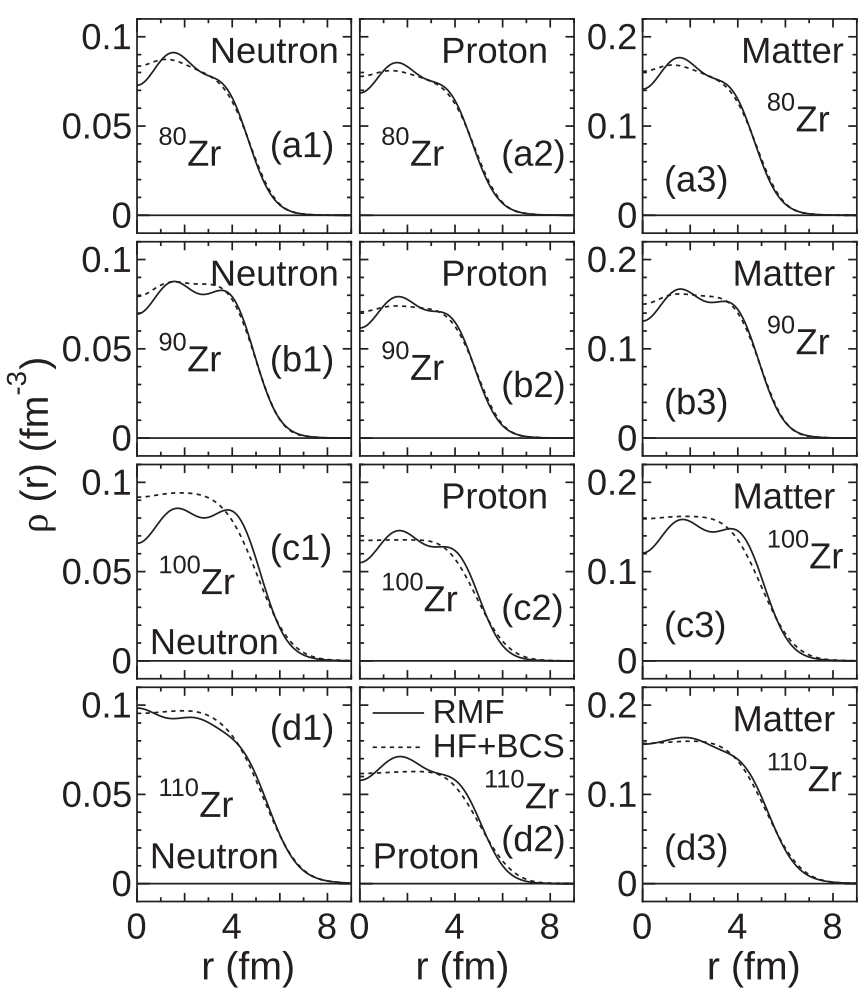

FIG. 4. Point-neutron, proton, and matter density distributions of (a) ${ }^{80} \mathrm{Zr}$, (b) ${ }^{90} \mathrm{Zr}$, (c) ${ }^{100} \mathrm{Zr}$, and (d) ${ }^{110} \mathrm{Zr}$.

rms radii of the point-proton, point-neutron, and point-matter densities for all the other nuclei.

We will discuss how those differences of the density profiles appear in the scattering observables later in Secs. IV D and IV D 3.

\section{FUNCTIONAL FORM OF MICROSCOPIC GLOBAL OPTICAL POTENTIAL}

Here we give the definition of a functional form of the present MGOP for the convenience of users. The central and spin-orbit components of the complex-valued NA SF potential are approximated in terms of a linear combination of 12-range Gaussian form as

$$
\begin{aligned}
& V_{\mathrm{CE}}^{\mathrm{MGOP}}(R) \equiv \sum_{i=1}^{12}\left\{\alpha_{\mathrm{CE}, i} \exp \left(-\frac{R^{2}}{\gamma_{\mathrm{CE}, i}^{2}}\right)\right\} \cong V_{\mathrm{CE}}(R), \\
& W_{\mathrm{CE}}^{\mathrm{MGOP}}(R) \equiv \sum_{i=1}^{12}\left\{\beta_{\mathrm{CE}, i} \exp \left(-\frac{R^{2}}{\gamma_{\mathrm{CE}, i}^{2}}\right)\right\} \cong W_{\mathrm{CE}}(R), \\
& V_{\mathrm{SO}}^{\mathrm{MGOP}}(R) \equiv \sum_{i=1}^{12}\left\{\alpha_{\mathrm{SO}, i} \exp \left(-\frac{R^{2}}{\gamma_{\mathrm{SO}, i}^{2}}\right)\right\} \cong V_{\mathrm{SO}}(R), \\
& W_{\mathrm{SO}}^{\mathrm{MGOP}}(R) \equiv \sum_{i=1}^{12}\left\{\beta_{\mathrm{SO}, i} \exp \left(-\frac{R^{2}}{\gamma_{\mathrm{SO}, i}^{2}}\right)\right\} \cong W_{\mathrm{SO}}(R)
\end{aligned}
$$



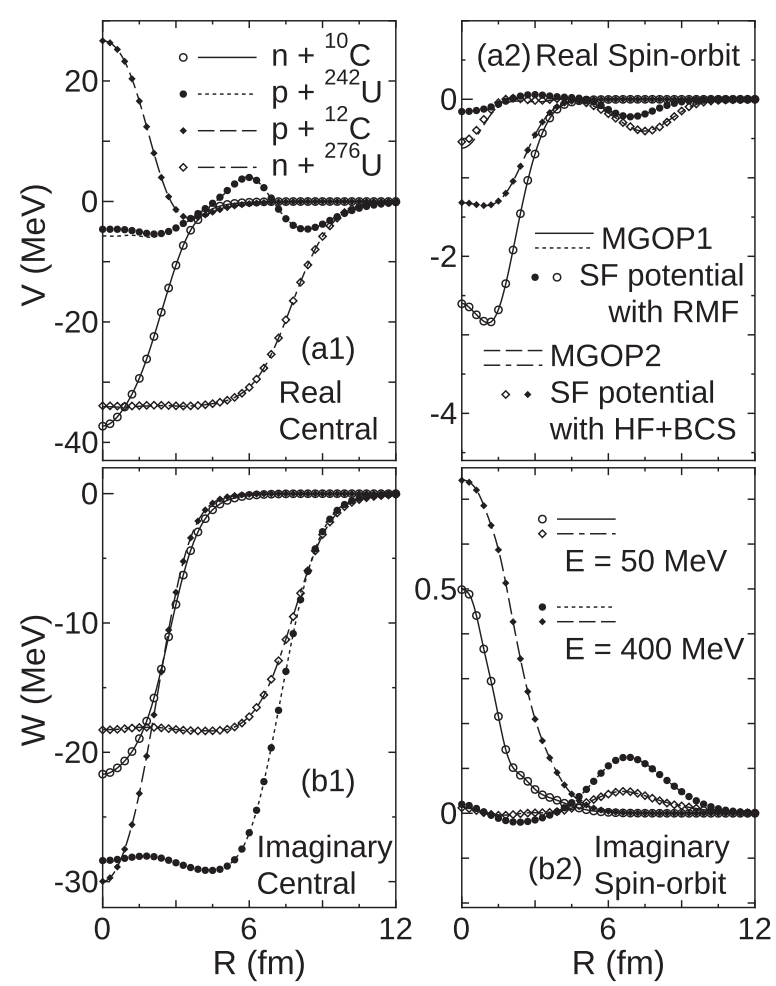

FIG. 5. Real parts of (a1) central and (a2) spin-orbit components and imaginary parts of (b1) central and (b2) spin-orbit components of the SF potentials. The original and 12-range Gaussian form factors are compared for $n+{ }^{10} \mathrm{C}$ at $E_{n}=50 \mathrm{MeV}$ and $p+{ }^{242} \mathrm{U}$ at $E_{p}=400 \mathrm{MeV}$ with the RMF densities (MGOP1); and $p+{ }^{12} \mathrm{C}$ at $E_{p}=400 \mathrm{MeV}$ and the $n+{ }^{276} \mathrm{U}$ at $E_{n}=50 \mathrm{MeV}$ with the $\mathrm{HF}+$ BCS densities (MGOP2).

with

$$
\begin{aligned}
\alpha_{\mathrm{CE}, i} & =\alpha_{\mathrm{CE}, i}\left(N, Z_{T}, A_{T}, E\right), \\
\beta_{\mathrm{CE}, i} & =\beta_{\mathrm{CE}, i}\left(N, Z_{T}, A_{T}, E\right), \\
\alpha_{\mathrm{SO}, i} & =\alpha_{\mathrm{SO}, i}\left(N, Z_{T}, A_{T}, E\right), \\
\beta_{\mathrm{SO}, i} & =\beta_{\mathrm{SO}, i}\left(N, Z_{T}, A_{T}, E\right), \\
\gamma_{i} & =\left(\frac{1}{4}\right)^{\frac{12-i}{11}}\left(0.5 A_{T}^{1 / 3}+1.5\right),
\end{aligned}
$$

where $Z_{T}, A_{T}$, and $E$ denote, the proton number of the target, the nucleon number of the target, and the incident energy of the nucleon, respectively. The values in Eq. (42) are chosen to ensure sufficient accuracy of the fitting to cover various potential form factors from light to heavy nuclear mass regions from 50 to $400 \mathrm{MeV}$ incident energies. The original and 12-range Gaussian form factors for the lightest and heaviest cases at $E=50$ and $400 \mathrm{MeV}$ are compared in Fig. 5. The MGOP1 and MGOP2 denote the MGOP obtained with the RMF and HF + BCS densities, respectively. We see that the original and 12-range Gaussian form factors are almost identical for light to heavy targets at low to high incident energies. The 12-range Gaussian form is sufficient to express various potential profiles generated from the SF procedure with the microscopic densities in a wide range of mass numbers and incident energies.
We calculate the SF potentials for the $p A$ and $n A$ systems with $Z_{T}=6-92$ and $A_{T}=10-276$ target nuclei at $E=50$, $60,70,80,90,100,120,140,160,180,200,300$, and $400 \mathrm{MeV}$. The parameter sets at other incident energies between 50 and $400 \mathrm{MeV}$ are obtained by the spline interpolation. All the parameter sets in Eqs. (38) to (41) are available on the website [43]. Since the total number of the parameters is huge about 2 million, for the convenience of users, we provide the program source "mgopN" on the website [43], which generates these parameter sets and construct the OMP for the $N A$ systems.

\section{RESULTS}

We here apply the present MGOP models to the $N A$ elastic scattering. The relativistic kinematics is used to compute the cross sections. Since the complex $G$-matrix is constructed in the infinite nuclear matter, the strength of the imaginary part needs to be adjusted when we apply it to a finite nuclear system because these level densities are quite different. Thus we introduce the incident-energy-dependent renormalization factor $N_{W}$ for the imaginary part of the OMP

$$
U=V_{\mathrm{CE}}^{\mathrm{MGOP}}+i N_{W} W_{\mathrm{CE}}^{\mathrm{MGOP}}+\left(V_{\mathrm{SO}}^{\mathrm{MGOP}}+i N_{W} W_{\mathrm{SO}}^{\mathrm{MGOP}}\right) \boldsymbol{\ell} \cdot \boldsymbol{\sigma} .
$$

The $N_{w}$ value is determined so as to reproduce the total reaction cross section data from 50 to $400 \mathrm{MeV}$ :

$$
N_{W}=0.5+E / 1000 .
$$

The validity of this choice will be examined in comparison with experimental data in the following sections.

To guide a reader, we give an outline of what we present in this section.

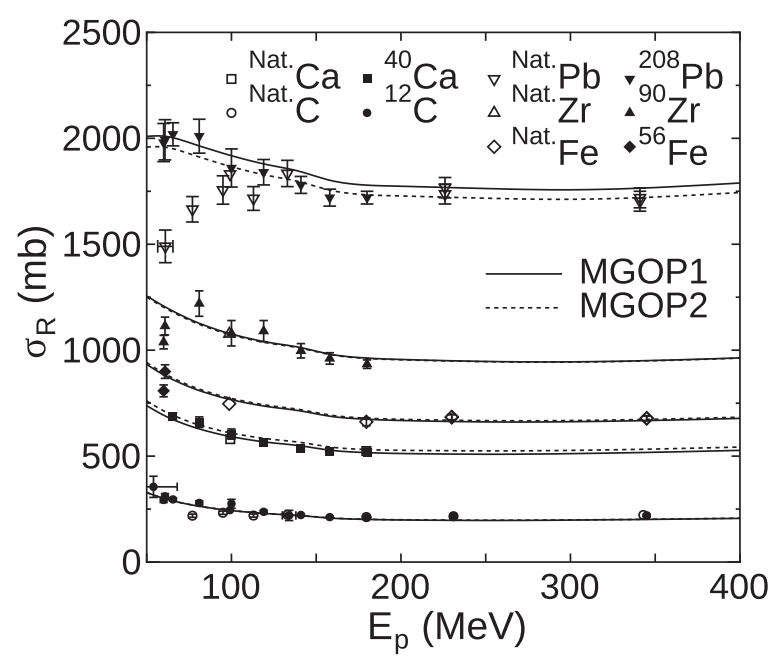

FIG. 6. Total reaction cross sections of $p+{ }^{12} \mathrm{C},{ }^{40} \mathrm{Ca},{ }^{56} \mathrm{Fe},{ }^{90} \mathrm{Zr}$, and ${ }^{208} \mathrm{~Pb}$ systems as a function of incident proton energy $E_{p}$. The RMF (MGOP1) and HF + BCS (MGOP2) densities are employed to construct the SF potential, respectively. The experimental data for ${ }^{\text {Nat. }} \mathrm{C}$ (natural carbon), ${ }^{12} \mathrm{C}$, Nat. $\mathrm{Ca}$ (natural calcium), ${ }^{40} \mathrm{Ca}$, ${ }^{\text {Nat. }} \mathrm{Fe}$ (natural iron), ${ }^{56} \mathrm{Fe},{ }^{\text {Nat. }} \mathrm{Zr}$ (natural zirconium), ${ }^{90} \mathrm{Zr}$, ${ }^{\text {Nat. }} \mathrm{Pb}$ (natural lead), and ${ }^{208} \mathrm{~Pb}$ targets are taken from Refs. [74-85]. 


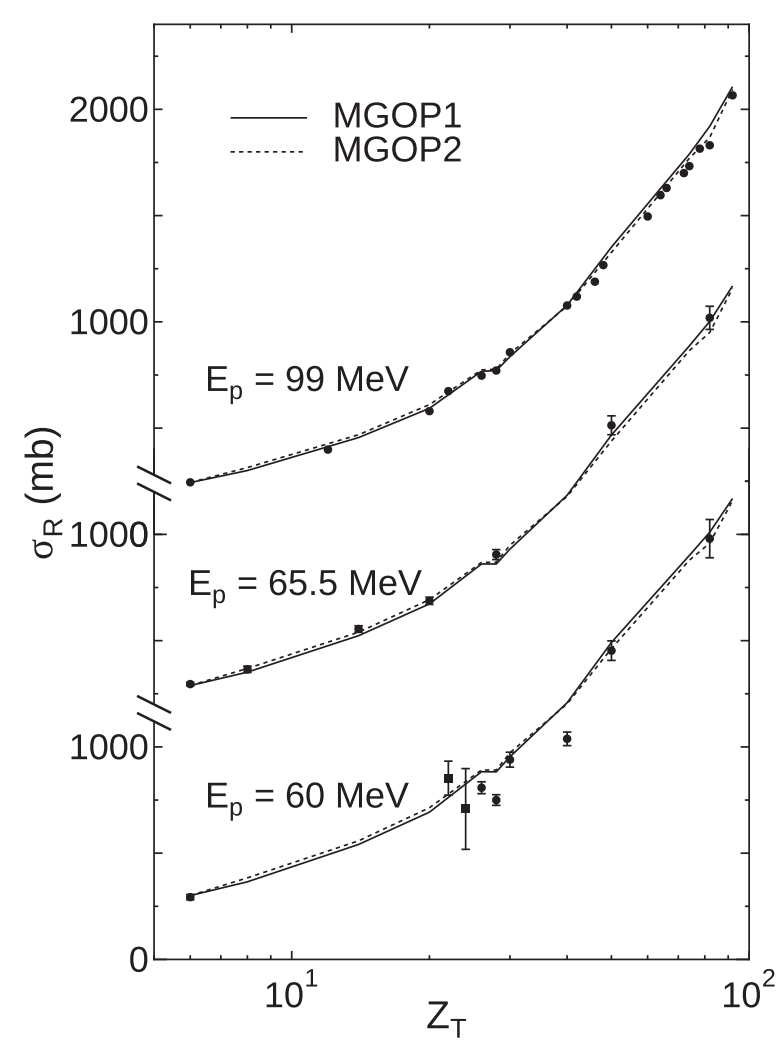

FIG. 7. Total reaction cross sections at $E_{p}=60,65.5$, and $99 \mathrm{MeV}$ as a function of the proton number of a target nucleus $Z_{T}$ calculated with the MGOP1 and MGOP2 models. See text for more details. The experimental data are taken from Refs. [74,81,82,84].

(1) (p.14) Sec. IV A. Total reaction cross sections As mentioned, the incident-energy dependence of the $N_{W}$ value is determined by the total reaction cross sections. We obtain overall agreement with the measured total reaction cross sections of $p A$ and $n A$ systems with the choice of $N_{W}$ in Eq. (44).

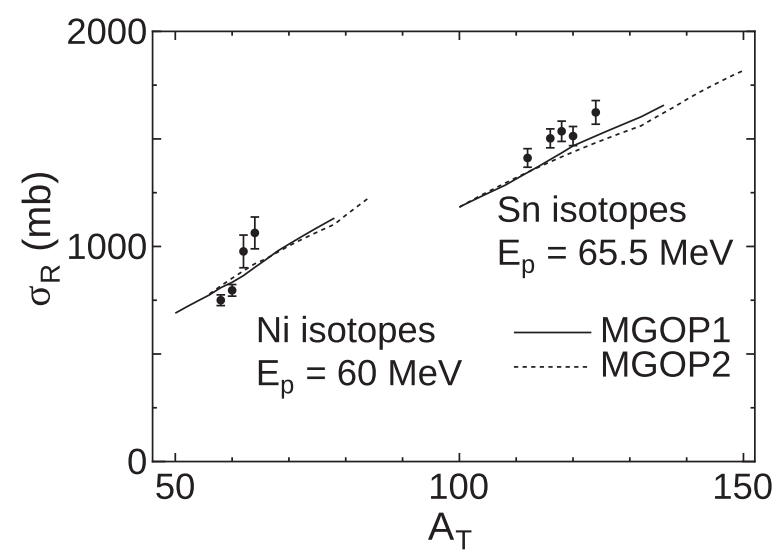

FIG. 8. Total reaction cross sections of $p+\mathrm{Ni}$ and $p+\mathrm{Sn}$ systems at $E_{p}=60$ and $65.5 \mathrm{MeV}$, respectively, calculated with the MGOP1 and MGOP2 models. The experimental data for ${ }^{58-64} \mathrm{Ni}$ and ${ }^{112-124} \mathrm{Sn}$ targets are taken from Refs. [74,80,82,84].

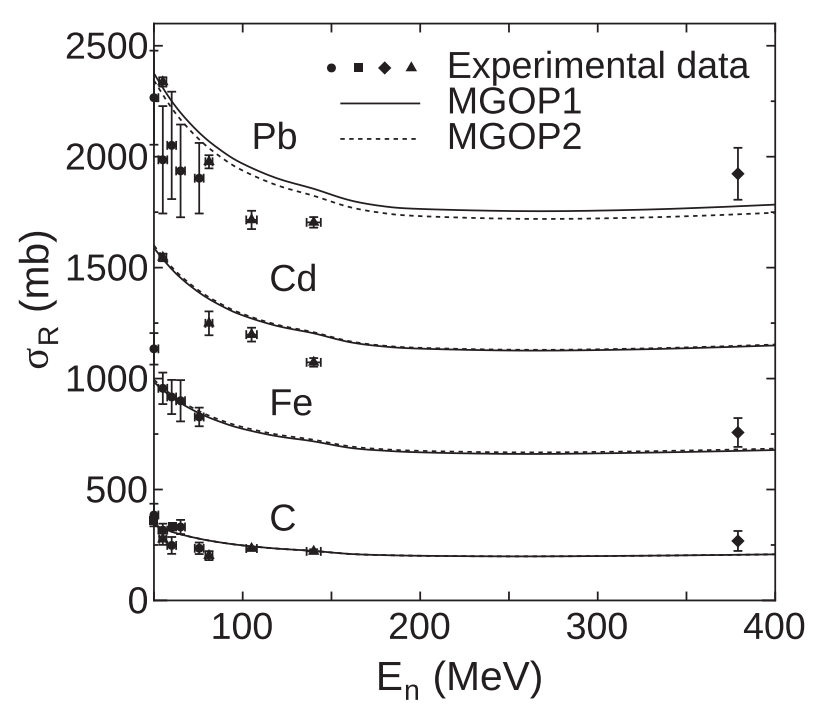

FIG. 9. Total reaction cross sections of $n+{ }^{12} \mathrm{C},{ }^{56} \mathrm{Fe},{ }^{114} \mathrm{Cd}$, and ${ }^{208} \mathrm{~Pb}$ systems as a function of incident neutron energy $E_{n}$ calculated with the MGOP1 and MGOP2 models. Experimental data for ${ }^{\text {Nat. }} \mathrm{C}$, ${ }^{\text {Nat. }} \mathrm{Fe}$, ${ }^{\text {Nat. }} \mathrm{Cd}$ (natural cadmium), and ${ }^{\text {Nat. }} \mathrm{Pb}$ targets are taken from Refs. [74,86-90].

(2) (p.16) Sec. IV B. Total neutron cross sections The present MGOP models are applied to total neutron cross sections. We show that the experimental data of ${ }^{12} \mathrm{C},{ }^{40} \mathrm{Ca},{ }^{90} \mathrm{Zr},{ }^{184} \mathrm{~W},{ }^{208} \mathrm{~Pb}$, and ${ }^{238} \mathrm{U}$ are reproduced.

(3) (p.16) Sec. IV C. Neutron-nucleus elastic scattering

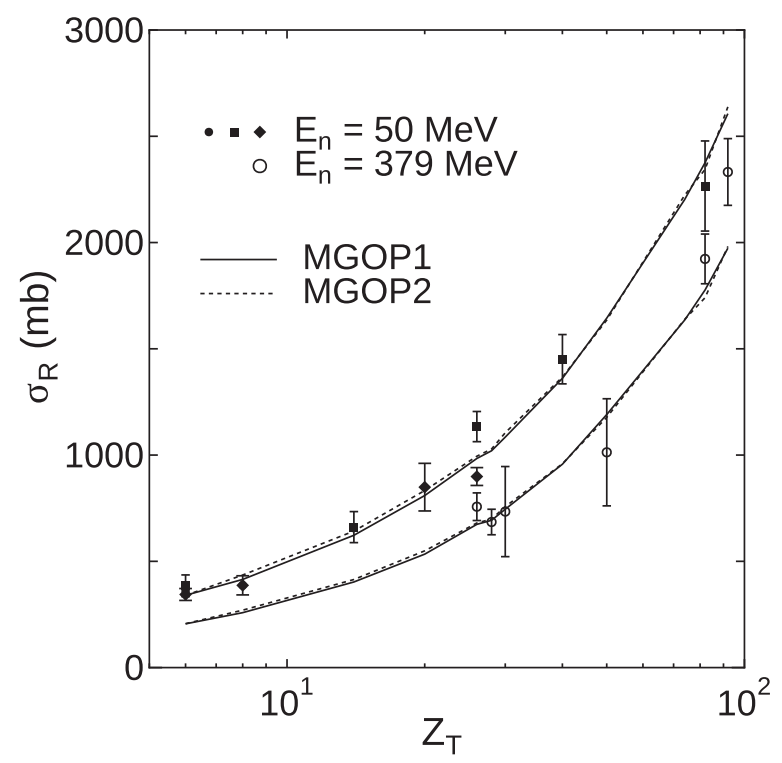

FIG. 10. Total reaction cross sections of $n A$ systems at $E_{n}=50$ and $379 \mathrm{MeV}$ as a function of the proton number of a target nucleus $Z_{T}$ calculated with the MGOP1 and MGOP2 models. See text for more details. The experimental data are taken from Refs. [74,87-90]. 


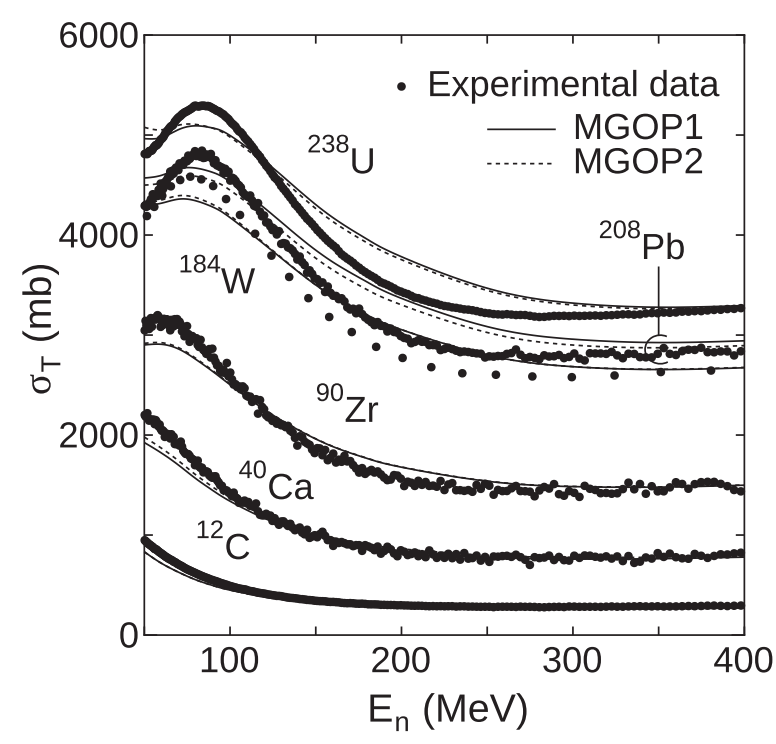

FIG. 11. Total neutron cross sections of $n A$ systems at $E_{n}=50$ and $400 \mathrm{MeV}$ as a function of the incident neutron energy $E_{n}$, calculated with the MGOP1 and MGOP2 models. The experimental data are taken from Refs. [74,91-93].

The present MGOP models are applied to $n A$ elastic scattering. We show that the existing experimental data are well reproduced.

(4) (p.17) Sec. IV D. Proton-nucleus elastic scattering

The present MGOP models are also applied to $p A$ elastic scattering. This section is decomposed into the following three subsections:

(p.17) Sec. IV D 1. Proton-nucleus elastic-scattering cross sections at $65 \mathrm{MeV}$

(p.18) Sec. IV D 2. Incident energy dependence of proton-nucleus elastic-scattering cross sections

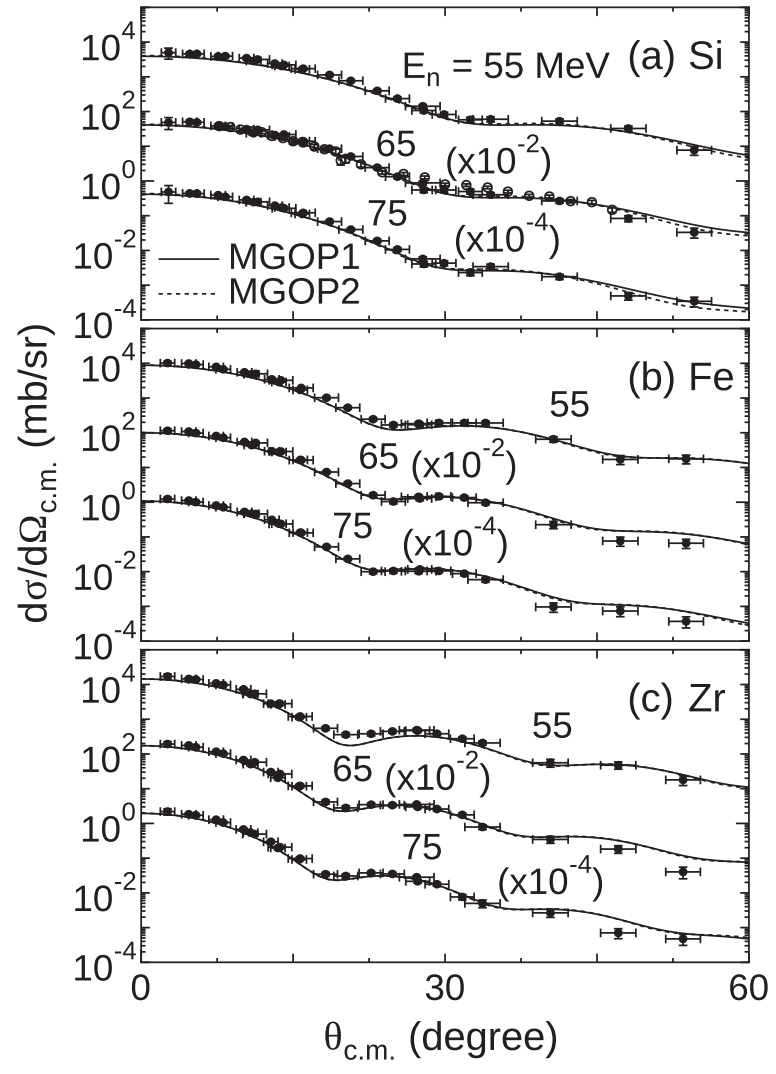

FIG. 13. Elastic-scattering cross sections of (a) $n+{ }^{28} \mathrm{Si}$, (b) ${ }^{56} \mathrm{Fe}$, and (c) ${ }^{90} \mathrm{Zr}$ systems at $E_{n}=55,65$, and $75 \mathrm{MeV}$. The experimental data for natural $\mathrm{Si}, \mathrm{Fe}$, and $\mathrm{Zr}$ targets are taken from Refs. [74,95].

(p.20) Sec. IV D 3. Proton-nucleus elastic-scattering cross sections for unstable nuclei.
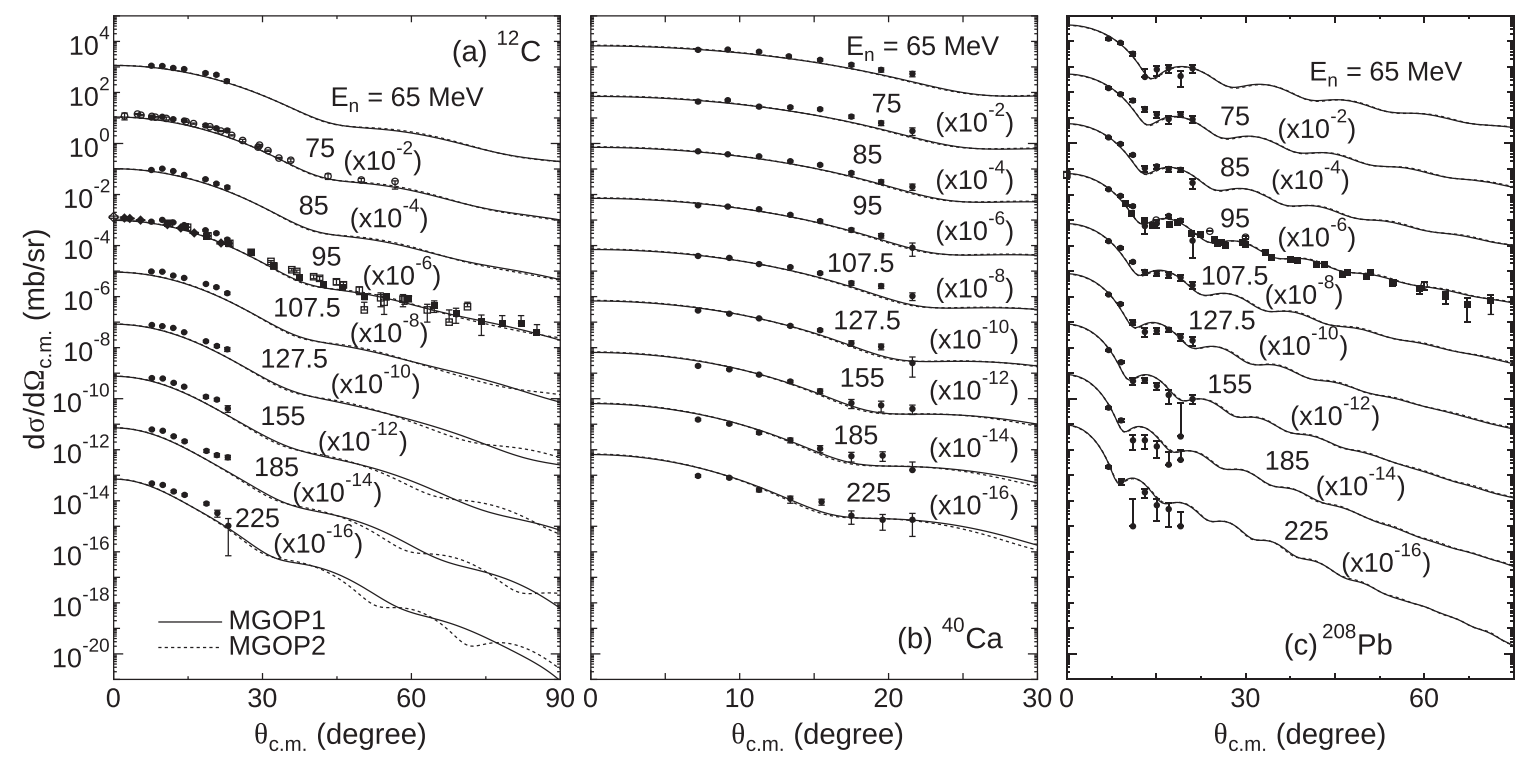

FIG. 12. Elastic-scattering cross sections of (a) $n+{ }^{12} \mathrm{C}$, (b) ${ }^{40} \mathrm{Ca}$, and (c) ${ }^{208} \mathrm{~Pb}$ systems at $E_{n}=65-225 \mathrm{MeV}$ with the MGOP1 (RMF) and MGOP2 (HF + BCS) models. The experimental data are taken from Refs. [74,94-100]. 


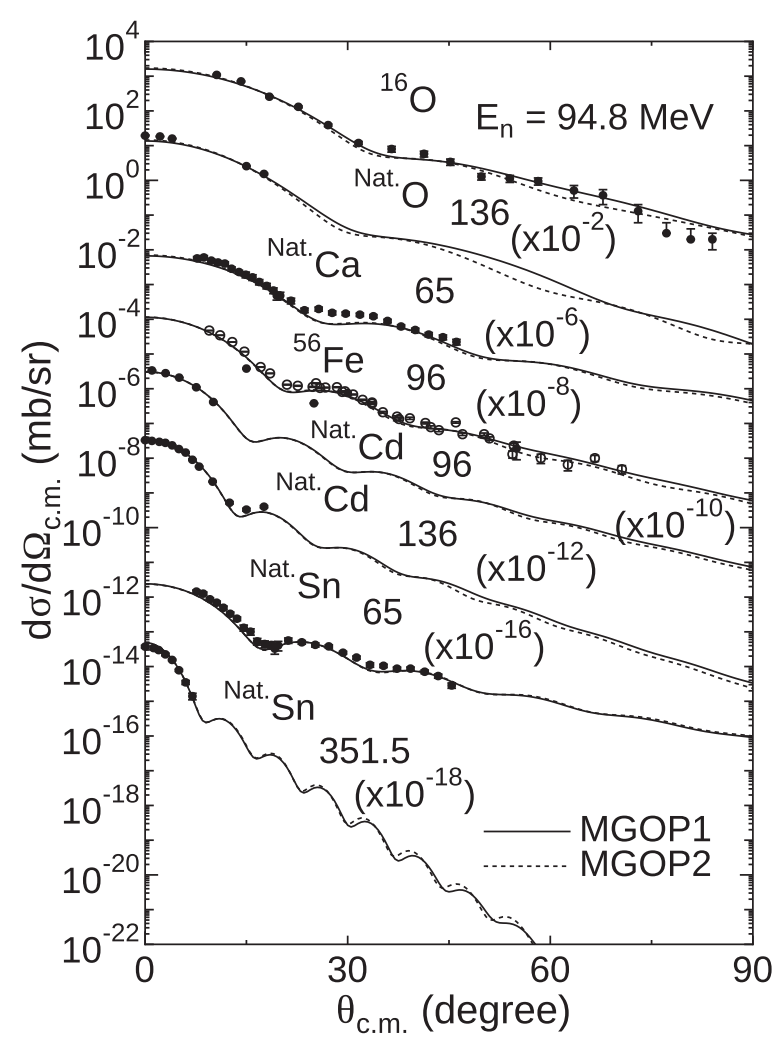

FIG. 14. Elastic-scattering cross sections of $n+{ }^{16} \mathrm{O},{ }^{40} \mathrm{Ca},{ }^{56} \mathrm{Fe}$, ${ }^{140} \mathrm{Cd}$, and ${ }^{120} \mathrm{Sn}$ systems at $E_{n}=65-351.5 \mathrm{MeV}$. The experimental data for ${ }^{16} \mathrm{O},{ }^{56} \mathrm{Fe}$, natural $\mathrm{O}, \mathrm{Ca}, \mathrm{Cd}$, and $\mathrm{Sn}$ targets are taken from Refs. [74,94,98-100,102-104].

\section{A. Total reaction cross sections}

Let us first examine the $N_{W}$ value obtained in Eq. (44) for the total reaction cross sections $\left(\sigma_{R}\right)$ of the $p A$ systems which are summarized in the following three figures.

(1) Figure 6: Total reaction cross sections of $p+{ }^{12} \mathrm{C}$, ${ }^{40} \mathrm{Ca},{ }^{56} \mathrm{Fe},{ }^{90} \mathrm{Zr}$, and ${ }^{208} \mathrm{~Pb}$ systems as a function of the incident proton energy $E_{p}$.

(2) Figure 7: Total reaction cross sections of $p+{ }^{12} \mathrm{C},{ }^{16} \mathrm{O}$, ${ }^{24} \mathrm{Mg},{ }^{28} \mathrm{Si},{ }^{40} \mathrm{Ca},{ }^{48} \mathrm{Ti},{ }^{50} \mathrm{Ti},{ }^{52} \mathrm{Cr},{ }^{56} \mathrm{Fe},{ }^{58} \mathrm{Ni},{ }^{64} \mathrm{Zn}$, ${ }^{68} \mathrm{Zn},{ }^{90} \mathrm{Zr},{ }^{98} \mathrm{Mo},{ }^{106} \mathrm{Pd},{ }^{114} \mathrm{Cd},{ }^{120} \mathrm{Sn},{ }^{142} \mathrm{Nd},{ }^{158} \mathrm{Gd}$, ${ }^{164} \mathrm{Dy},{ }^{180} \mathrm{Hf},{ }^{184} \mathrm{~W},{ }^{194} \mathrm{Pt},{ }^{208} \mathrm{~Pb}$, and ${ }^{238} \mathrm{U}$ systems at $E_{p}=60,65.5$, and $99 \mathrm{MeV}$ as a function of the proton number of a target nucleus $Z_{T}$.

(3) Figure 8: Total reaction cross sections of $p+\mathrm{Ni}$ and $p+$ Sn targets at $E_{p}=60$ and $65.5 \mathrm{MeV}$ as a function of the mass number of a target nucleus $A_{T}$.

As one can see from these figures, both the cross sections with the MGOP1 (RMF; solid line) and MGOP2 (HF + BCS; dotted line) models fairly well reproduce the experimental data. The $N_{W}$ value assumed in Eq. (44) works well in describing the energy dependence of the total reaction cross sections of the $p A$ systems in a wide range of the mass numbers.

We also check the validity of $N_{W}$ for the $n A$ total reaction cross sections. Figure 9 shows the calculated total reaction

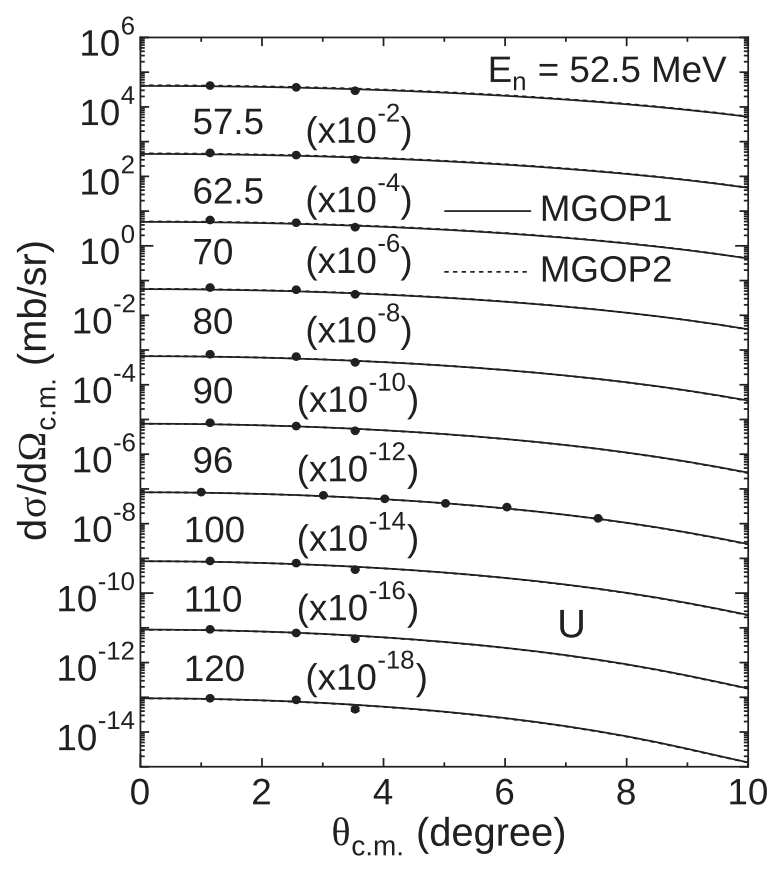

FIG. 15. Elastic-scattering cross sections of $n+{ }^{238} \mathrm{U}$ system at $E_{n}=52.5-120 \mathrm{MeV}$. The experimental data for a natural $\mathrm{U}$ target are taken from Refs. [74,94,105].

cross sections of $n+{ }^{12} \mathrm{C},{ }^{56} \mathrm{Fe},{ }^{114} \mathrm{Cd}$, and ${ }^{208} \mathrm{~Pb}$ systems as a function of the incident neutron energy $E_{n}$. To see the massnumber dependence, we plot, in Fig. 10, the calculated total reaction cross sections of $n+{ }^{12} \mathrm{C},{ }^{16} \mathrm{O},{ }^{28} \mathrm{Si},{ }^{40} \mathrm{Ca},{ }^{56} \mathrm{Fe},{ }^{58} \mathrm{Ni}$, ${ }^{64} \mathrm{Zn},{ }^{90} \mathrm{Zr},{ }^{114} \mathrm{Cd},{ }^{120} \mathrm{Sn},{ }^{208} \mathrm{~Pb}$, and ${ }^{238} \mathrm{U}$ systems at $E_{n}=50$ and $379 \mathrm{MeV}$. As displayed in Fig. 9, the calculated cross sections well reproduce the experimental data for the natural carbon $\left({ }^{\text {Nat. }} \mathrm{C}\right.$ ) and iron ( ${ }^{\text {Nat. }} \mathrm{Fe}$ ) targets for $E_{n} \leqslant 150 \mathrm{MeV}$. Though we see good agreement of the theoretical calculations with the natural cadmium ( $\left.{ }^{\text {Nat. }} \mathrm{Cd}\right)$ and lead $\left({ }^{\mathrm{Nat}} \mathrm{Pb}\right)$ data around $50 \mathrm{MeV}$, the calculated cross sections overestimate these data around $100 \mathrm{MeV}$. The theoretical values slightly underestimate the experimental data of ${ }^{\text {Nat. }} \mathrm{C}$, Nat. $\mathrm{Fe}$, and ${ }^{\text {Nat. }} \mathrm{Pb}$ around $400 \mathrm{MeV}$, whereas they agree well with the experimental data for natural nickel ( $\left.{ }^{\text {Nat. }} \mathrm{Ni}\right)$, zirconium ( ${ }^{\text {Nat. }} \mathrm{Zn}$ ), and tin ( ${ }^{\text {Nat. }} \mathrm{Sn}$ ) targets at $E_{n}=379 \mathrm{MeV}$ as shown in Fig. 10.

The MGOP1 and MGOP2 show almost identical results for the NA total reaction cross sections despite the fact that the RMF and HF + BCS give quite different charge radii and point-nucleon density distributions as shown in Figs. 2, 3, and 4 because the total reaction cross section of the $N A$ scattering is determined mostly by the rms radius of the matter density and is rather insensitive to the density distribution [67]. In fact, the difference of the rms radii obtained with the RMF and $\mathrm{HF}+\mathrm{BCS}$ models is within $2 \%$.

The renormalization factor assumed in Eq. (44) gives a reasonable description of the available experimental data of the total reaction cross sections of the $p A$ and $n A$ systems. Hereafter, we use this energy-dependent renormalization factor to evaluate the total neutron cross sections and NA elasticscattering cross sections. 

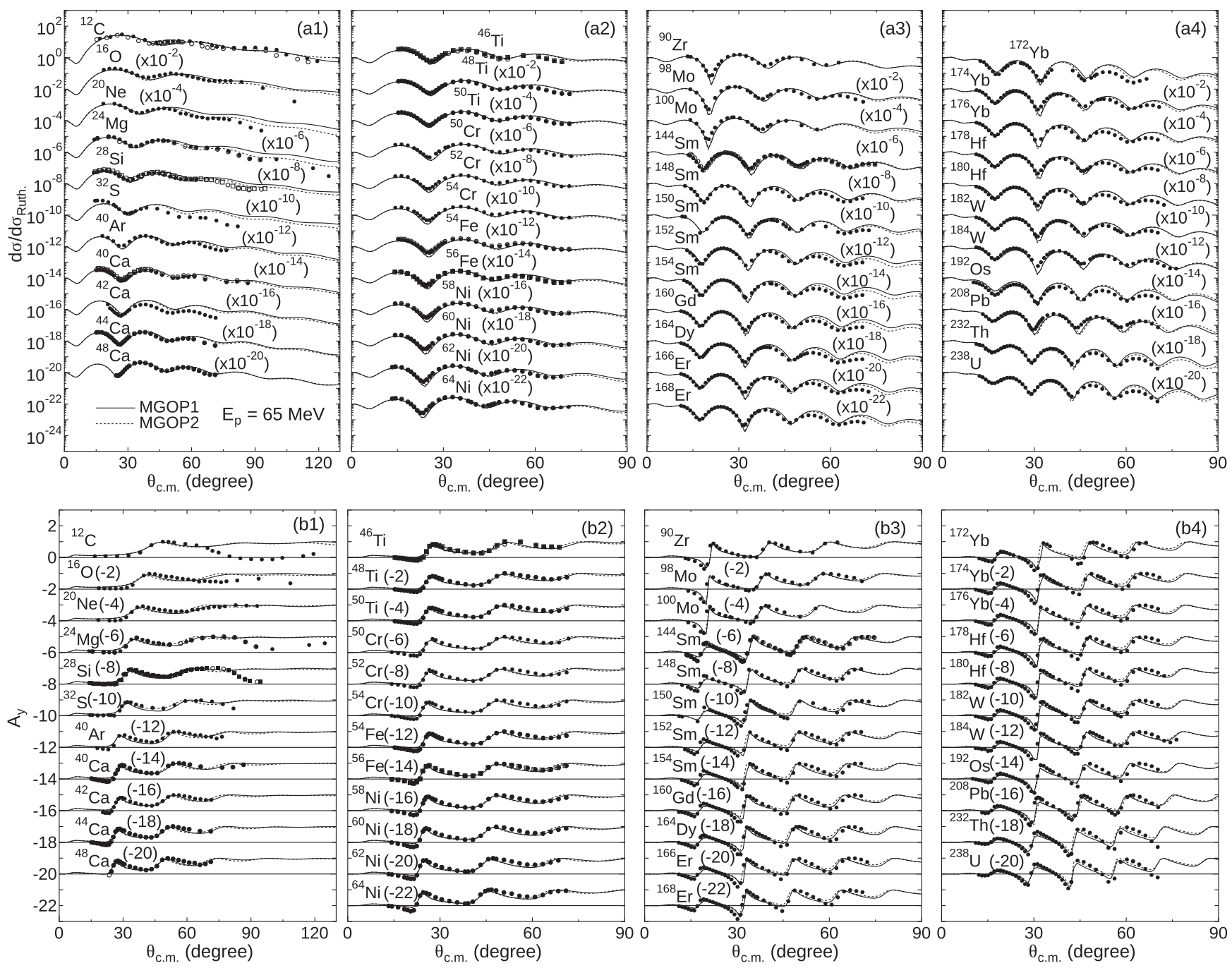

FIG. 16. (a) Elastic-scattering cross sections in the Rutherford ratio and (b) analyzing power of $p A$ systems with $Z_{T}=6-92$ at $E_{p}=$ $65 \mathrm{MeV}$. The experimental data are taken from Refs. [74,106-120].

\section{B. Total neutron cross sections}

We here test the MGOP1 and MGOP2 for the $n A$ total cross sections. Figure 11 shows the calculated total neutron cross sections of $n+{ }^{12} \mathrm{C},{ }^{40} \mathrm{Ca},{ }^{90} \mathrm{Zr},{ }^{184} \mathrm{~W},{ }^{208} \mathrm{~Pb}$, and ${ }^{238} \mathrm{U}$ systems as a function of the incident neutron energy, $E_{n}$. The ${ }^{40} \mathrm{Ca}$, ${ }^{90} \mathrm{Zr}$, and ${ }^{208} \mathrm{~Pb}$ nuclei are well known as a spherical shape, while the ${ }^{12} \mathrm{C},{ }^{184} \mathrm{~W}$, and ${ }^{238} \mathrm{U}$ nuclei are well deformed. As displayed in Fig. 11, the calculated cross sections well reproduce the experimental data for the ${ }^{12} \mathrm{C},{ }^{40} \mathrm{Ca}$, and ${ }^{90} \mathrm{Zr}$ targets. The theoretical values show some deviations for the ${ }^{184} \mathrm{~W},{ }^{208} \mathrm{~Pb}$, and ${ }^{238} \mathrm{U}$ targets. These may be improved by taking the channel coupling effect into account, especially at the low incident energies. Again, the MGOP1 and MGOP2 show almost identical results for the $N A$ total cross sections.

\section{Neutron-nucleus elastic scattering}

In this section, we present the results of the $n A$ elastic scattering.

Figure 12 displays the elastic-scattering cross sections of $n+{ }^{12} \mathrm{C},{ }^{40} \mathrm{Ca}$, and ${ }^{208} \mathrm{~Pb}$ systems at $E_{n}=65-225 \mathrm{MeV}$. The solid and dotted curves are the calculated results with the MGOP1 (RMF) and MGOP2 (HF + BCS), respectively. Both the MGOP models well reproduce the experimental data up to backward angles, showing only slight difference between the two models at the backward angles in the high energy regions $E_{n} \gtrsim 100 \mathrm{MeV}$ of the $n+{ }^{12} \mathrm{C}$ scattering.

We also calculate the elastic-scattering cross sections of $n+{ }^{12} \mathrm{C}$ and ${ }^{208} \mathrm{~Pb}$ systems, and confirm that the calculated cross sections well reproduce the experimental data for natural $\mathrm{C}$ and $\mathrm{Pb}$ targets $[74,94,95,101-104]$. The interested readers are referred to the website [43] for these results.

Let us see the cross sections of medium-mass nuclei. Figure 13 shows the elastic-scattering cross sections of $n+{ }^{28} \mathrm{Si}$, ${ }^{56} \mathrm{Fe}$, and ${ }^{90} \mathrm{Zr}$ systems at $E_{n}=55-75 \mathrm{MeV}$. The calculated cross sections with the two MGOP models perfectly reproduce the experimental data for the ${ }^{\text {Nat. }} \mathrm{Si}$, ${ }^{\text {Nat. }} \mathrm{Fe}$, and ${ }^{\mathrm{Nat}} \mathrm{Zr}$ targets up to backward angles.

We calculate the cross sections from light to mediumheavy nuclei. Figure 14 displays the elastic-scattering cross sections of $n+{ }^{16} \mathrm{O},{ }^{40} \mathrm{Ca},{ }^{56} \mathrm{Fe},{ }^{140} \mathrm{Cd}$, and ${ }^{120} \mathrm{Sn}$ targets at $E_{n}=65-351.5 \mathrm{MeV}$. The experimental data are the ones for 

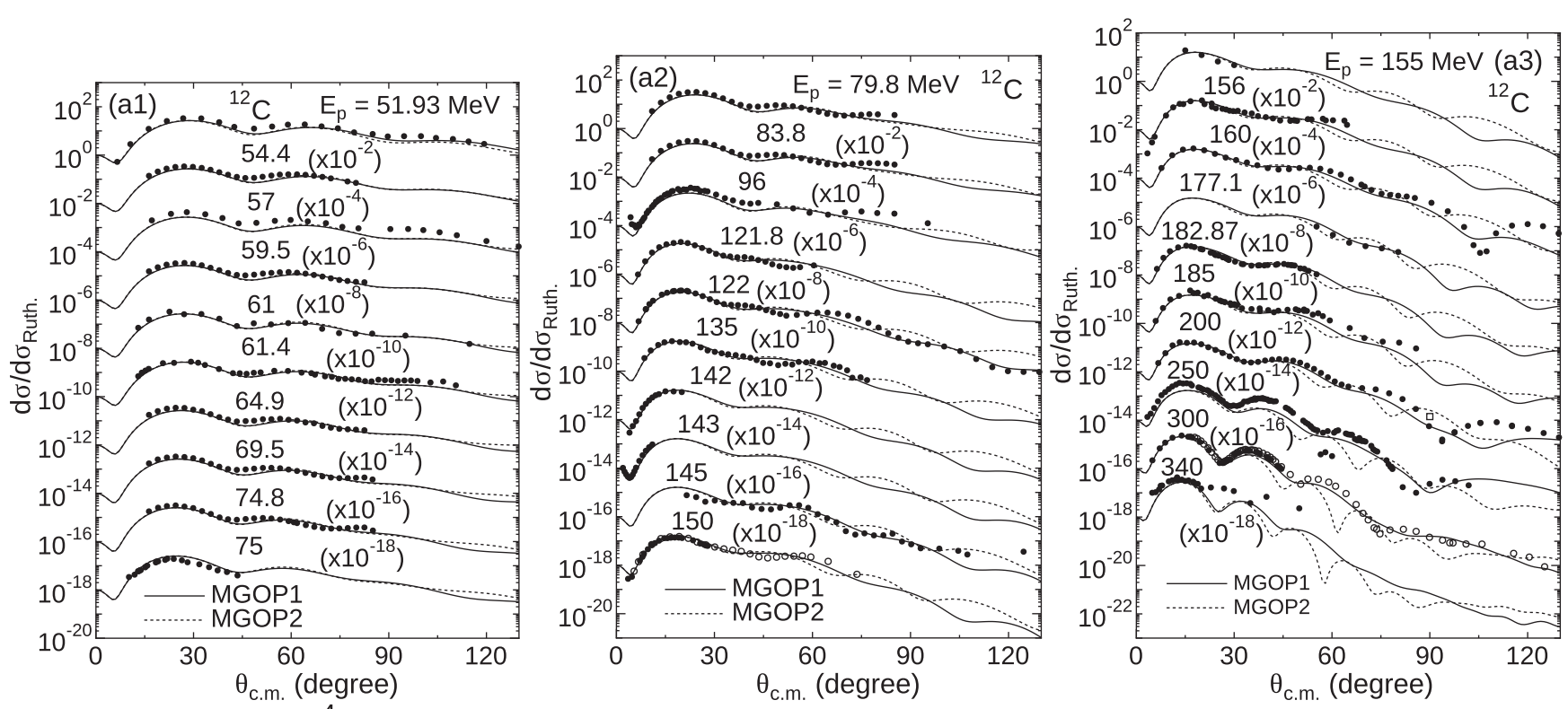

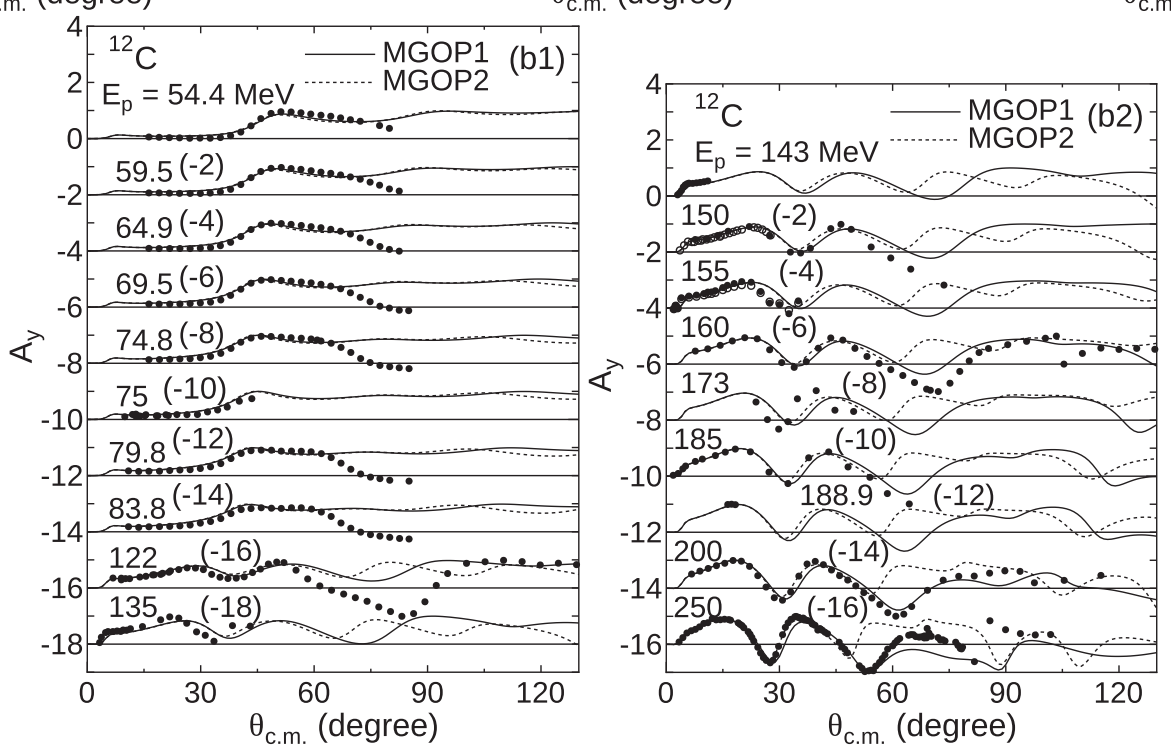

FIG. 17. (a) Elastic-scattering cross sections in the Rutherford ratio at $E_{p}=51.93-340 \mathrm{MeV}$ and (b) analyzing power at $E_{p}=$ 54.4-250 MeV of $p+{ }^{12} \mathrm{C}$ system. The experimental data are taken from Refs. [74,121-149].

${ }^{16} \mathrm{O}$, ${ }^{\text {Nat. }} \mathrm{O}$, ${ }^{\text {Nat. }} \mathrm{Ca},{ }^{56} \mathrm{Fe}$, ${ }^{\text {Nat. }} \mathrm{Cd}$, and ${ }^{\text {Nat. }}$ Sn targets. Again, the experimental data are well reproduced with the two MGOP models.

At the end of this section, we calculate the cross sections of $n+{ }^{238} \mathrm{U}$ system which involves the heaviest target nucleus presented in this paper. Figure 15 plots the $n+{ }^{238} \mathrm{U}$ elasticscattering cross sections at $E_{n}=52.5-120 \mathrm{MeV}$. Although the data are available only up to $8^{\circ}$, the results with the two MGOP models are identical and successfully reproduce the experimental data for a natural $\mathrm{U}$ target.

Those comparisons we made confirm the validity of the MGOP models for the $n A$ elastic scattering.

\section{Proton-nucleus elastic scattering}

\section{Proton-nucleus elastic-scattering cross sections at $65 \mathrm{MeV}$}

In this section, we compare the MGOP results with experimental data for the proton elastic-scattering cross sections and analyzing power focusing on the mass number dependence. We first examine the $p A$ elastic-scattering cross sections at $65 \mathrm{MeV}$, where a number of experimental data are available.

Figure 16 plots the $p A$ elastic-scattering cross section and analyzing power at $E_{p}=65 \mathrm{MeV}$ with the following target nuclei:

(1) $Z_{T}=6-20:{ }^{12} \mathrm{C},{ }^{16} \mathrm{O},{ }^{20} \mathrm{Ne},{ }^{24} \mathrm{Mg},{ }^{28} \mathrm{Si},{ }^{32} \mathrm{~S},{ }^{40} \mathrm{Ar}$, ${ }^{40} \mathrm{Ca},{ }^{42} \mathrm{Ca},{ }^{44} \mathrm{Ca}$, and ${ }^{48} \mathrm{Ca}$;

(2) $Z_{T}=22-28:{ }^{46} \mathrm{Ti},{ }^{48} \mathrm{Ti},{ }^{50} \mathrm{Ti},{ }^{50} \mathrm{Cr},{ }^{52} \mathrm{Cr},{ }^{54} \mathrm{Cr}{ }^{54} \mathrm{Fe}$, ${ }^{56} \mathrm{Fe},{ }^{58} \mathrm{Ni},{ }^{60} \mathrm{Ni},{ }^{62} \mathrm{Ni}$, and ${ }^{64} \mathrm{Ni}$;

(3) $Z_{T}=40-68:{ }^{90} \mathrm{Zr},{ }^{98} \mathrm{Mo},{ }^{100} \mathrm{Mo},{ }^{144} \mathrm{Sm},{ }^{148} \mathrm{Sm},{ }^{150} \mathrm{Sm}$, ${ }^{152} \mathrm{Sm},{ }^{154} \mathrm{Sm},{ }^{160} \mathrm{Gd},{ }^{164} \mathrm{Dy},{ }^{166} \mathrm{Er}$, and ${ }^{168} \mathrm{Er}$;

(4) $Z_{T}=70-92:{ }^{172} \mathrm{Yb},{ }^{174} \mathrm{Yb},{ }^{176} \mathrm{Yb},{ }^{178} \mathrm{Hf},{ }^{180} \mathrm{Hf},{ }^{182} \mathrm{~W}$, ${ }^{184} \mathrm{~W},{ }^{192} \mathrm{Os},{ }^{208} \mathrm{~Pb},{ }^{232} \mathrm{Th}$, and ${ }^{238} \mathrm{U}$.

The present MGOP models successfully describe the elastic scattering processes and reproduce the experimental data 
up to backward angles, which confirm the validity of the present MGOP models with various targets $\left(Z_{T}=6-92\right)$ for the $p A$ elastic scattering at $65 \mathrm{MeV}$. However, at a closer look, we see some discrepancy in the analyzing power at the backward angles between the theory and experiment for some light target nuclei, ${ }^{12} \mathrm{C},{ }^{24} \mathrm{Mg}$, and ${ }^{28} \mathrm{Si}$. We find the slight differences between the results with the RMF (MGOP1) and $\mathrm{HF}+\mathrm{BCS}$ (MGOP2) models for $Z_{T}=10-16$, where their density distributions are quite different as shown in Fig. 3. More detailed discussions will be made by examining the incident energy dependence of the cross sections in the Sec. IV D 3.

\section{Incident energy dependence of proton-nucleus elastic-scattering cross sections}

Here we investigate the validity of the present MGOP models with the cross sections calculated at various incident energies.

Figure 17 shows the elastic-scattering cross sections at $E_{p}=51.93-340 \mathrm{MeV}$ and analyzing power at $E_{p}=$ $54.4-250 \mathrm{MeV}$ of $p+{ }^{12} \mathrm{C}$ system. The elastic-scattering cross sections are given in the Rutherford ratio. We see the present MGOP models describe these scattering processes very well. With increasing the incident energy, the difference between the cross sections with the MGOP1 (RMF; solid line) and MGOP2 (HF + BCS; dotted line) becomes more apparent. Such a high energy beam is advantageous to look into the details of the nuclear density. We find the MGOP1 shows better agreement with the $p+{ }^{12} \mathrm{C}$ scattering cross section data, especially at the high-incident energies.

Figure 18 displays the $p+{ }^{16} \mathrm{O}$ elastic-scattering cross sections at $E_{p}=61-317.4 \mathrm{MeV}$ and the analyzing power at $E_{p}=$ $135-317.4 \mathrm{MeV}$. We also see good agreement of the theoretical calculations with the experimental data up to backward angles. Here we find that the difference of the MGOP models with the RMF and HF + BCS densities clearly appears above $E_{p}=200 \mathrm{MeV}$ at the backward angles as already observed in the ${ }^{12} \mathrm{C}$ case. From this global analysis of the $p+{ }^{16} \mathrm{O}$ scattering the RMF model appears to be better for describing the elastic-scattering cross section data, whereas the HF + BCS model shows better agreement with the analyzing power at $317.4 \mathrm{MeV}$.

For the medium mass nuclei $\left(A_{T}=24-82\right)$, we plot the following.

(1) Figure 19: Elastic-scattering cross sections at $E_{p}=51.9-400 \mathrm{MeV}$ and analyzing power at $E_{p}=55-317.7 \mathrm{MeV}$ of $p+{ }^{24} \mathrm{Mg},{ }^{28} \mathrm{Si},{ }^{30} \mathrm{Si},{ }^{32} \mathrm{~S}$, and ${ }^{34} \mathrm{~S}$ systems.

(2) Figure 20: Elastic-scattering cross sections at $E_{p}=51.93-400 \mathrm{MeV}$, analyzing power at $E_{p}=75-400 \mathrm{MeV}$ of $p+{ }^{40} \mathrm{Ca}$ and ${ }^{48} \mathrm{Ca}$ systems, and spin-rotation function $Q$ at $E_{p}=65-300 \mathrm{MeV}$ of $p+{ }^{40} \mathrm{Ca}$ system.

(3) Figure 21: Elastic-scattering cross sections of $p+$ ${ }^{46} \mathrm{Ti},{ }^{48} \mathrm{Ti},{ }^{50} \mathrm{Ti},{ }^{50} \mathrm{Cr},{ }^{54} \mathrm{Fe},{ }^{56} \mathrm{Fe},{ }^{58} \mathrm{Ni},{ }^{60} \mathrm{Ni},{ }^{62} \mathrm{Ni},{ }^{66} \mathrm{Zn}$, ${ }^{68} \mathrm{Zn},{ }^{74} \mathrm{Se},{ }^{76} \mathrm{Se},{ }^{78} \mathrm{Se},{ }^{80} \mathrm{Se}$, and ${ }^{82} \mathrm{Se}$ systems at $E_{p}=$ 51.9-333 MeV; the analyzing power of $p+{ }^{54} \mathrm{Fe},{ }^{56} \mathrm{Fe}$, ${ }^{58} \mathrm{Ni}$, and ${ }^{60} \mathrm{Ni}$ systems at $E_{p}=60.2-295 \mathrm{MeV}$; and
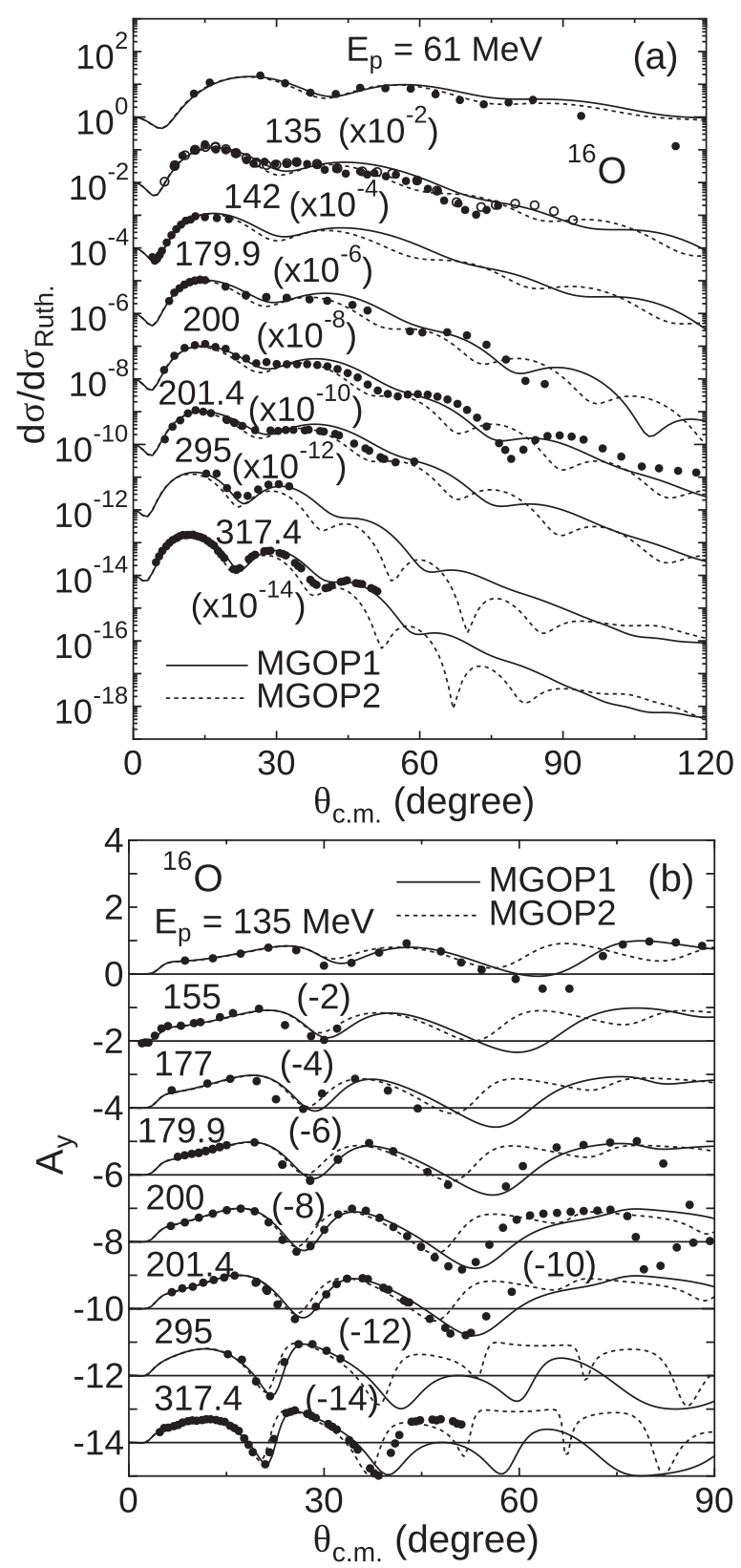

FIG. 18. (a) Elastic-scattering cross sections in the Rutherford ratio at $E_{p}=61-317.4 \mathrm{MeV}$ and (b) analyzing power at $E_{p}=$ $135-317.4 \mathrm{MeV}$ of $p+{ }^{16} \mathrm{O}$ system. The experimental data are taken from Refs. [74,124,125,128,134,150-156].

spin-rotation function $Q$ of $p+{ }^{58} \mathrm{Ni}$ system at $E_{p}=$ 200 and $300 \mathrm{MeV}$.

As displayed in Figs. 19 to 21, the calculated cross sections well reproduce the experimental data. We see from Fig. 19 the difference between the cross sections with the MGOP1 and MGOP2 models at the backward angles above $E_{p}=200 \mathrm{MeV}$ reflects the different density profiles as shown in Fig. 3. As all the calculated results shown in Figs. 19 to 21, the difference of the two MGOP models becomes clearer with increasing the incident energies: The present MGOPs give almost identical results at $E_{p} \leqslant 200 \mathrm{MeV}$, whereas the calculated results with 

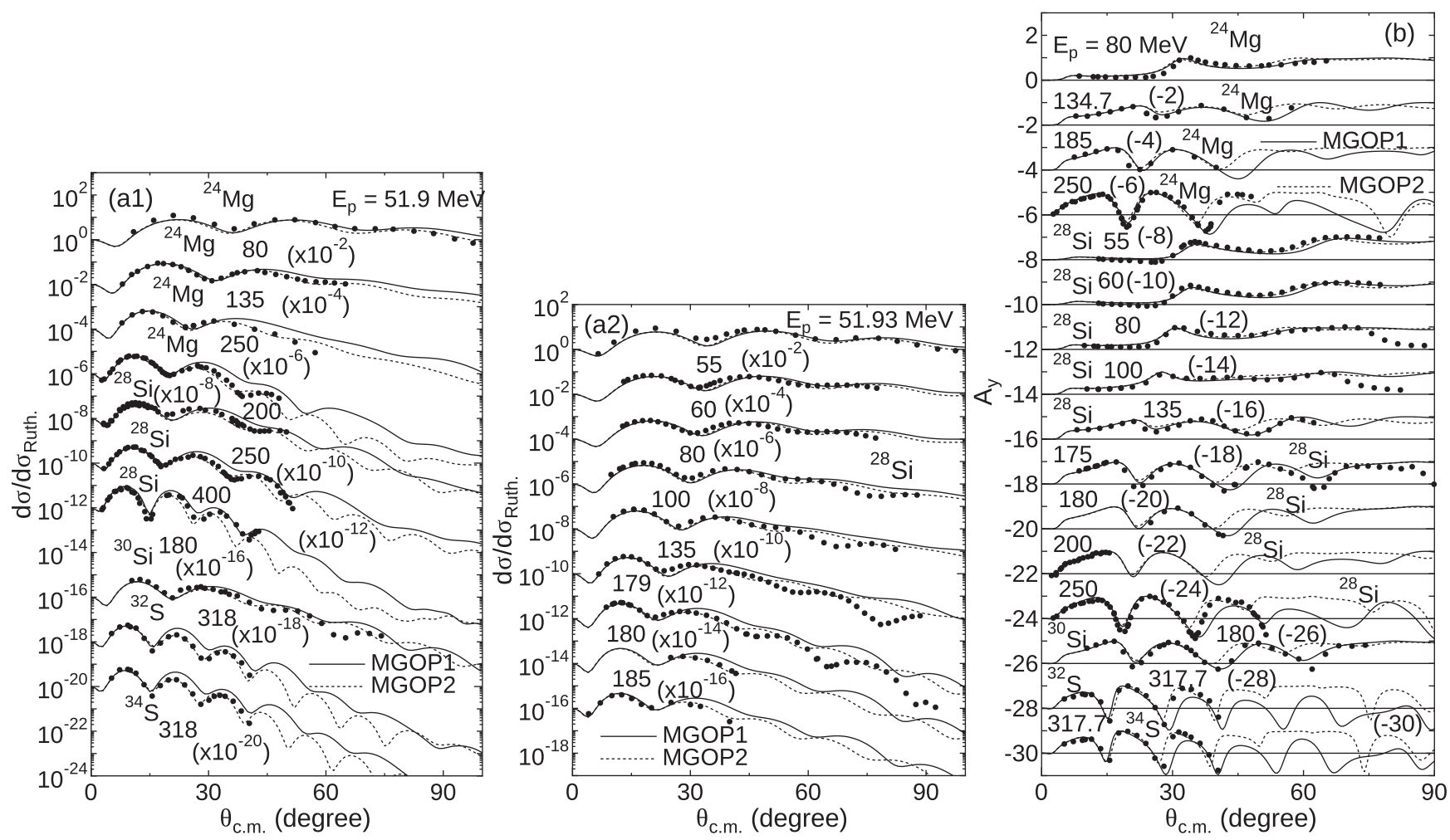

FIG. 19. (a) Elastic-scattering cross sections of $p+{ }^{24} \mathrm{Mg},{ }^{28} \mathrm{Si},{ }^{30} \mathrm{Si},{ }^{32} \mathrm{~S}$, and ${ }^{34} \mathrm{~S}$ systems at $E_{p}=51.9-400 \mathrm{MeV}$ in the Rutherford ratio and (b) analyzing power of $p+{ }^{24} \mathrm{Mg},{ }^{28} \mathrm{Si},{ }^{30} \mathrm{Si},{ }^{32} \mathrm{~S}$, and ${ }^{34} \mathrm{~S}$ systems at $E_{p}=55-317.7 \mathrm{MeV}$. The experimental data are taken from Refs. [74,112,137,157-166].

the two MGOP models deviate at $E_{p} \geqslant 200 \mathrm{MeV}$ in the backward angles. Measurements of these cross sections with such a high energy beam can distinguish the two theoretical models and will be useful to extract the information on the density profiles.

For heavier systems $\left(A_{T}=88-124\right)$, we plot the following:

(1) Figure 22: Elastic-scattering cross sections and analyzing power of $p+{ }^{88} \mathrm{Sr},{ }^{90} \mathrm{Zr}$, and ${ }^{92} \mathrm{Zr}$ systems at $E_{p}=$ 57.5-200.4 MeV. Spin-rotation function $Q$ of $p+{ }^{90} \mathrm{Zr}$ system at $65 \mathrm{MeV}$.

(2) Figure 23: Elastic-scattering cross sections of $p+$ ${ }^{100} \mathrm{Mo},{ }^{106} \mathrm{Pd},{ }^{108} \mathrm{Pd},{ }^{110} \mathrm{Pd},{ }^{112} \mathrm{Cd}$, and ${ }^{116} \mathrm{Sn}$ systems at $E_{p}=51-61.4 \mathrm{MeV}$.

(3) Figure 24: Elastic-scattering cross sections of $p+$ ${ }^{116} \mathrm{Sn},{ }^{118} \mathrm{Sn},{ }^{120} \mathrm{Sn},{ }^{122} \mathrm{Sn}$, and ${ }^{124} \mathrm{Sn}$ systems at $E_{p}=61.5-300 \mathrm{MeV}$; the analyzing power of $p+$ ${ }^{116} \mathrm{Sn},{ }^{118} \mathrm{Sn},{ }^{120} \mathrm{Sn},{ }^{122} \mathrm{Sn}$, and ${ }^{124} \mathrm{Sn}$ systems at $E_{p}=$ $103.5-300 \mathrm{MeV}$; and the spin-rotation function $Q$ of $p+{ }^{120} \mathrm{Sn}$ system at 200 and $300 \mathrm{MeV}$.

Both the MGOP models well reproduce the experimental data up to backward angles. No clear difference is found in the cross sections with the two MGOP models in those mass regions.

We show the results of heaviest systems $\left(A_{T}=148-208\right)$. Figure 25 displays the elastic-scattering cross sections of $p+{ }^{148} \mathrm{Sm},{ }^{154} \mathrm{Sm},{ }^{192} \mathrm{Os},{ }^{194} \mathrm{Pt},{ }^{198} \mathrm{Pt},{ }^{204} \mathrm{~Pb},{ }^{206} \mathrm{~Pb}$, and ${ }^{208} \mathrm{~Pb}$ systems at $E_{p}=61.4-400 \mathrm{MeV}$; the analyzing power of $p+$ ${ }^{192} \mathrm{Os},{ }^{194} \mathrm{Pt},{ }^{198} \mathrm{Pt},{ }^{204} \mathrm{~Pb},{ }^{206} \mathrm{~Pb}$, and ${ }^{208} \mathrm{~Pb}$ systems at $E_{p}=$
79.8-400 MeV; and the spin-rotation function $Q$ of $p+{ }^{208} \mathrm{~Pb}$ system at $E_{p}=65-300 \mathrm{MeV}$. As one can see from the figure, the calculated cross sections well reproduce the experimental data which show the validity of the present MGOP models for the heavy targets.

As demonstrated in this section, the present MGOP models successfully reproduce the numerous experimental data of the elastic-scattering cross sections, analyzing power, and spinrotation function $Q$ in a wide range of mass number and incident energies, which show the reliability of the present MGOP models. One of the advantages of the present approach is that one can obtain the OMP for unstable nuclear systems in a consistent manner. In the next section, though the experimental data are limited, we show the comparison to the theoretical cross sections for the unstable nuclei and the theoretical predictions for selected neutron-rich unstable nuclei.

\section{Proton-nucleus elastic-scattering cross sections for unstable nuclei}

Here we also test the MGOP1 and MGOP2 for the proton elastic-scattering cross sections of unstable nuclei, ${ }^{22} \mathrm{O},{ }^{24} \mathrm{O}$, ${ }^{56} \mathrm{Ni}$ where the experimental data are available. In addition, we show some results of the proton elastic-scattering cross section and the analyzing power involving the unstable nuclei far from the stability line. We select ${ }^{48} \mathrm{~S},{ }^{100} \mathrm{Zr}$, and ${ }^{110} \mathrm{Zr}$ because the two mean-field models give the quite different 

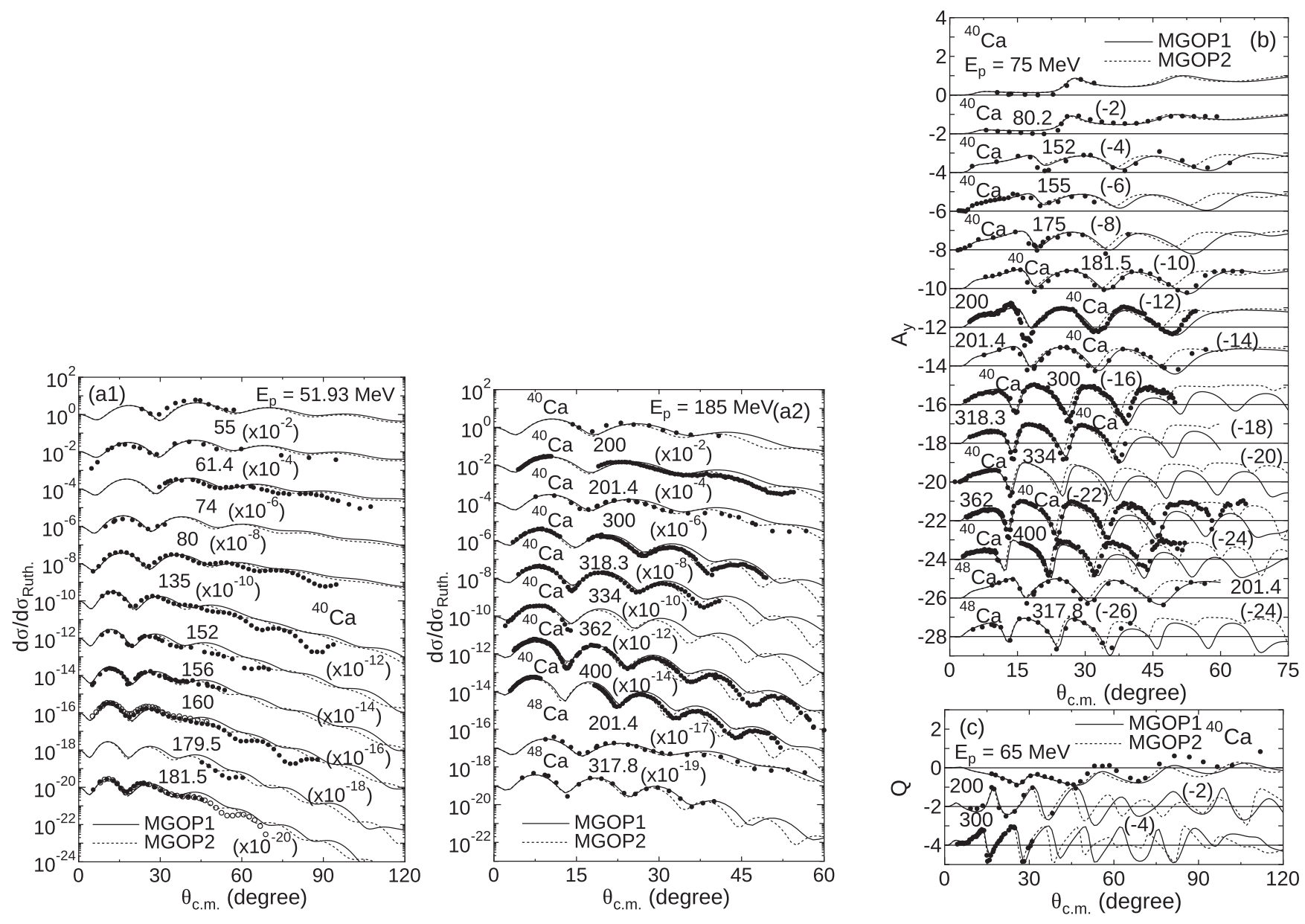

FIG. 20. (a) Elastic-scattering cross sections of $p+{ }^{40} \mathrm{Ca}$ and ${ }^{48} \mathrm{Ca}$ systems at $E_{p}=51.93-400 \mathrm{MeV}$ in the Rutherford ratio; (b) analyzing power of $p+{ }^{40} \mathrm{Ca}$ and ${ }^{48} \mathrm{Ca}$ systems at $E_{p}=75-400 \mathrm{MeV}$; and (c) spin-rotation function $Q$ of $p+{ }^{40} \mathrm{Ca}$ system at $E_{p}=65-300 \mathrm{MeV}$. The experimental data are taken from Refs. [4,32,33,74,124,125,127,132,133,135,137,155,159,166-173].
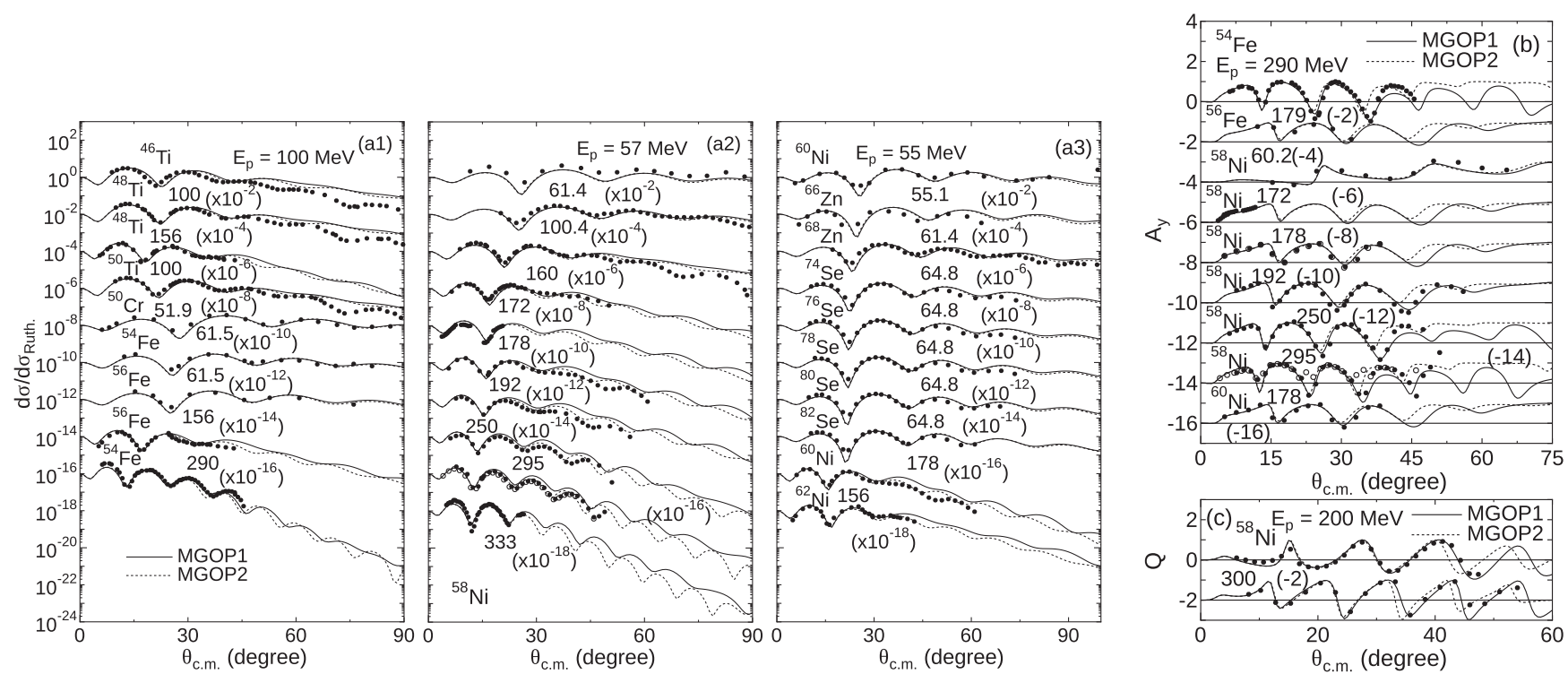

FIG. 21. (a) Elastic-scattering cross sections of $p+{ }^{46} \mathrm{Ti},{ }^{48} \mathrm{Ti},{ }^{50} \mathrm{Ti},{ }^{50} \mathrm{Cr},{ }^{54} \mathrm{Fe},{ }^{56} \mathrm{Fe},{ }^{58} \mathrm{Ni},{ }^{60} \mathrm{Ni},{ }^{62} \mathrm{Ni},{ }^{66} \mathrm{Zn},{ }^{68} \mathrm{Zn},{ }^{74} \mathrm{Se},{ }^{76} \mathrm{Se},{ }^{78} \mathrm{Se}$, ${ }^{80} \mathrm{Se}$, and ${ }^{82} \mathrm{Se}$ systems at $E_{p}=51.9-333 \mathrm{MeV}$ in the Rutherford ratio; (b) analyzing power of $p+{ }^{54} \mathrm{Fe},{ }^{56} \mathrm{Fe},{ }^{58} \mathrm{Ni}$, and ${ }^{60} \mathrm{Ni}$ systems at $E_{p}=60.2-295 \mathrm{MeV}$; and (c) spin-rotation function $Q$ of $p+{ }^{58} \mathrm{Ni}$ at $E_{p}=200$ and $300 \mathrm{MeV}$. The experimental data are taken from Refs. [74,129,133-135,137,168,173-188]. 

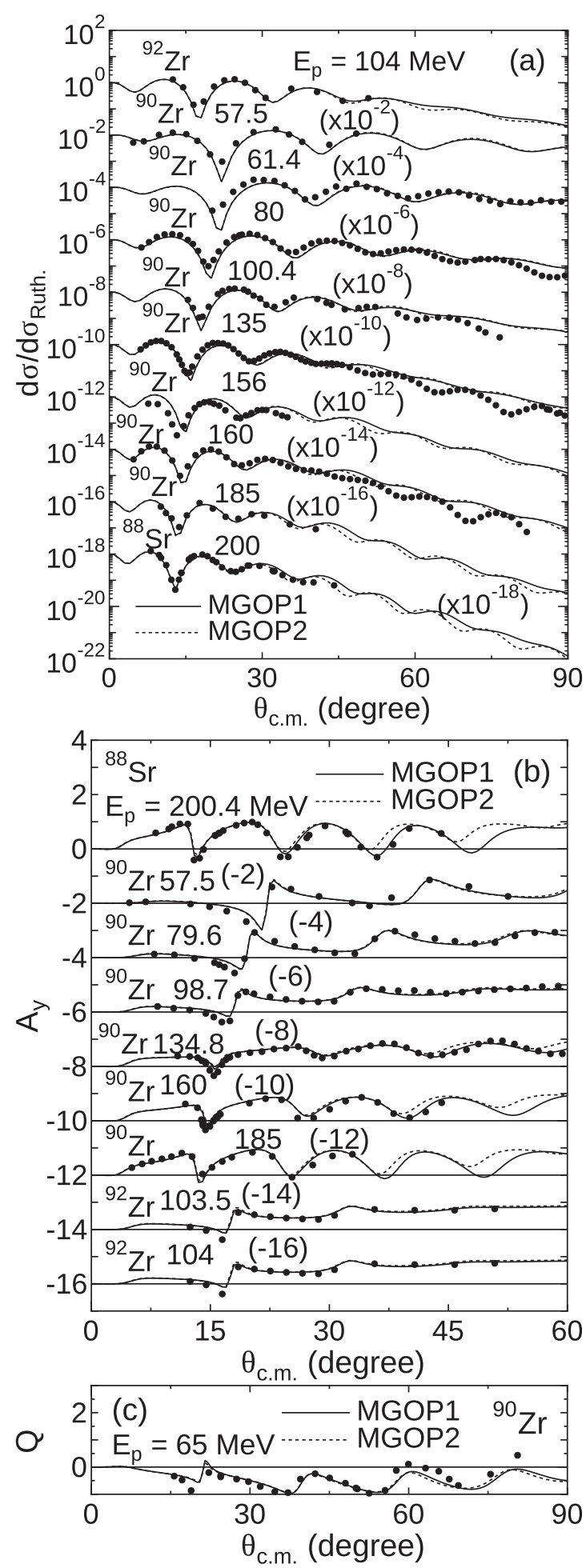

FIG. 22. (a) Elastic-scattering cross sections in the Rutherford ratio at $E_{p}=57.5-200 \mathrm{MeV}$; (b) analyzing power at $E_{p}=57.5-200.4 \mathrm{MeV}$ of $p+{ }^{88} \mathrm{Sr},{ }^{90} \mathrm{Zr}$, and ${ }^{92} \mathrm{Zr}$ systems; and (c) Spin-rotation function $Q$ at $E_{p}=65 \mathrm{MeV}$ of $p+{ }^{90} \mathrm{Zr}$ system. The experimental data are taken from Refs. [4,32,74,133,135,159,178,189-192].

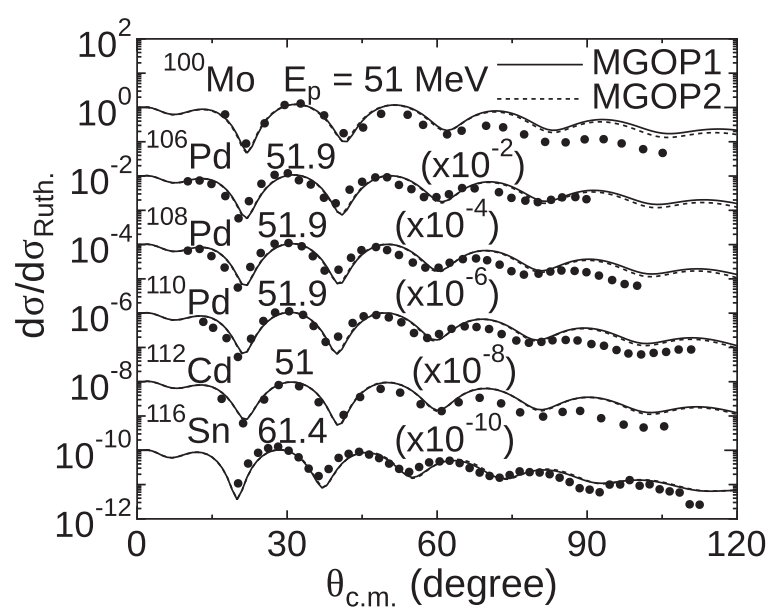

FIG. 23. Elastic-scattering cross sections of $p+{ }^{106} \mathrm{Pd}$, ${ }^{108} \mathrm{Pd},{ }^{110} \mathrm{Pd},{ }^{112} \mathrm{Cd}$, and ${ }^{116} \mathrm{Sn}$ systems in the Rutherford ratio at $E_{p}=51-61.4 \mathrm{MeV}$. The experimental data are taken from Refs. [74,133,193,194].

prediction of the density distributions as shown in Figs. 3 and 4.

Figure 26 plots the elastic-scattering cross sections and the analyzing power of the proton scattering of $p+{ }^{22} \mathrm{O},{ }^{24} \mathrm{O}$, and ${ }^{56} \mathrm{Ni}$ systems at $E_{p}=46.6-285 \mathrm{MeV}$. Here we should note that the proton elastic-scattering cross sections of the $p+{ }^{22} \mathrm{O}$ system at $E_{p}=46.6 \mathrm{MeV}$ are obtained with the MGOP1 and MGOP2 at $50 \mathrm{MeV}$. The MGOP1 (RMF) and MGOP2 $(\mathrm{HF}+\mathrm{BCS})$ well reproduce the experimental data for $p+$ ${ }^{22} \mathrm{O}$ system at $E_{p}=46.6 \mathrm{MeV}$ and $p+{ }^{56} \mathrm{Ni}$ system at $E_{p}=$ $101 \mathrm{MeV}$ The difference between the cross sections with the MGOP1 and MGOP2 models appears in $p+{ }^{22} \mathrm{O}$ system at $E_{p}=285 \mathrm{MeV}$ and $p+{ }^{24} \mathrm{O}$ system at $E_{p}=264 \mathrm{MeV}$.

It is worthwhile to apply the present MGOP model to such unstable nuclei, where experimental data are not available. Figure 27 plots the elastic-scattering cross sections and the analyzing power of the proton scattering of $p+{ }^{48} \mathrm{~S}$ system at $E_{p}=100-400 \mathrm{MeV}$. The more the incident energy increases, the more apparent the difference between the cross sections with the MGOP1 and MGOP2 models becomes.

Figure 28 plots the elastic-scattering cross sections and the analyzing power of $p+{ }^{100} \mathrm{Zr}$ and ${ }^{110} \mathrm{Zr}$ systems at $E_{p}=$ $100-400 \mathrm{MeV}$. Again, we clearly see the difference of the two MGOP models in these scattering observables with increasing the incident energy. The $p A$ elastic scattering at such high incident energies can be a good probe to explore the density profiles of unstable nuclei, especially in the surface region. See also a recent work focusing on the nuclear "diffuseness" using a high-energy NA scattering [209]. These isotopes are known to be well deformed [210-212]. It is also interesting to consider the channel coupling effect due to the nuclear deformation.

\section{SUMMARY}

We constructed new microscopic global optical potentials (MGOPs) for the nucleon-nucleus $(N A)$ systems at $E=$ $50-400 \mathrm{MeV}$ using the single-folding (SF) model with the 

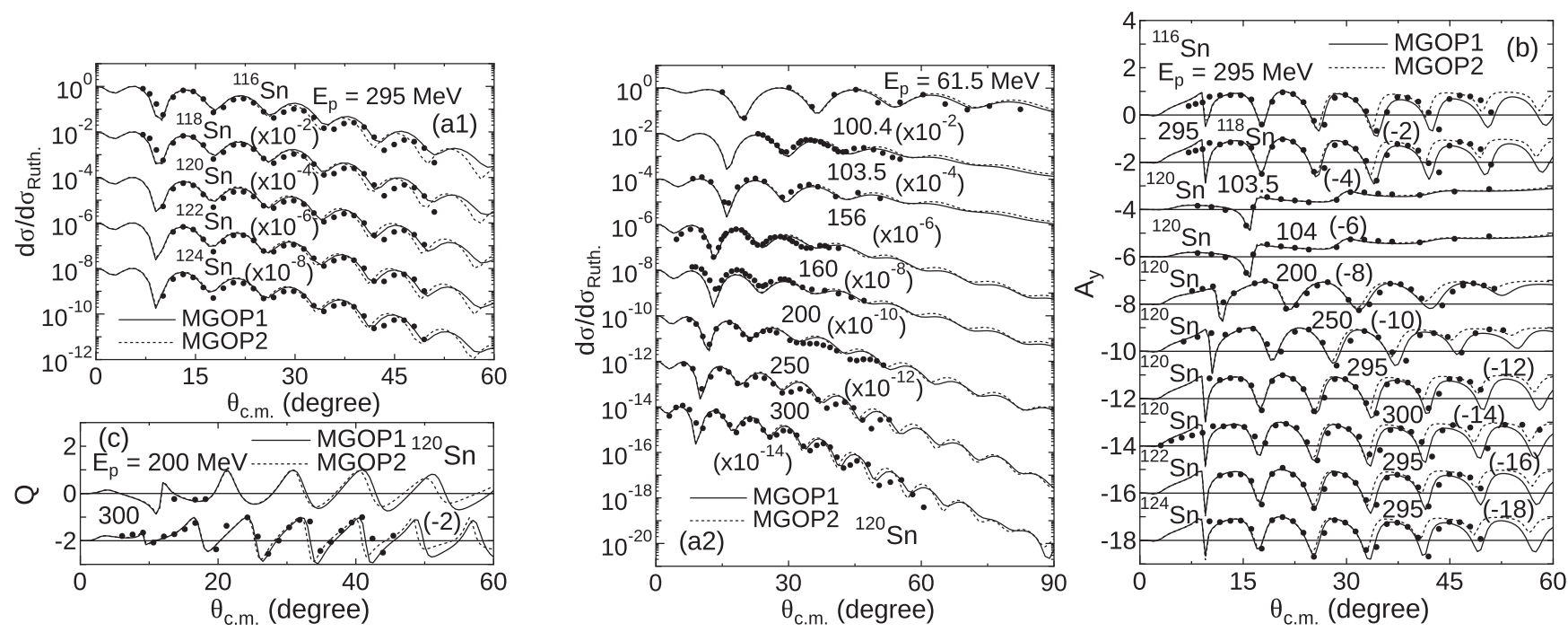

FIG. 24. (a) Elastic-scattering cross sections in the Rutherford ratio at $E_{p}=61.5-300 \mathrm{MeV}$; (b) analyzing power at $E_{p}=103.5-300 \mathrm{MeV}$ of $p+{ }^{116} \mathrm{Sn},{ }^{118} \mathrm{Sn},{ }^{120} \mathrm{Sn},{ }^{122} \mathrm{Sn}$, and ${ }^{124} \mathrm{Sn}$ systems; and (c) Spin-rotation function $Q$ at $E_{p}=200$ and $300 \mathrm{MeV}$ of $p+{ }^{120} \mathrm{Sn}$ system. The experimental data are taken from Refs. [74,134,135,159,168,178,186,191,195].
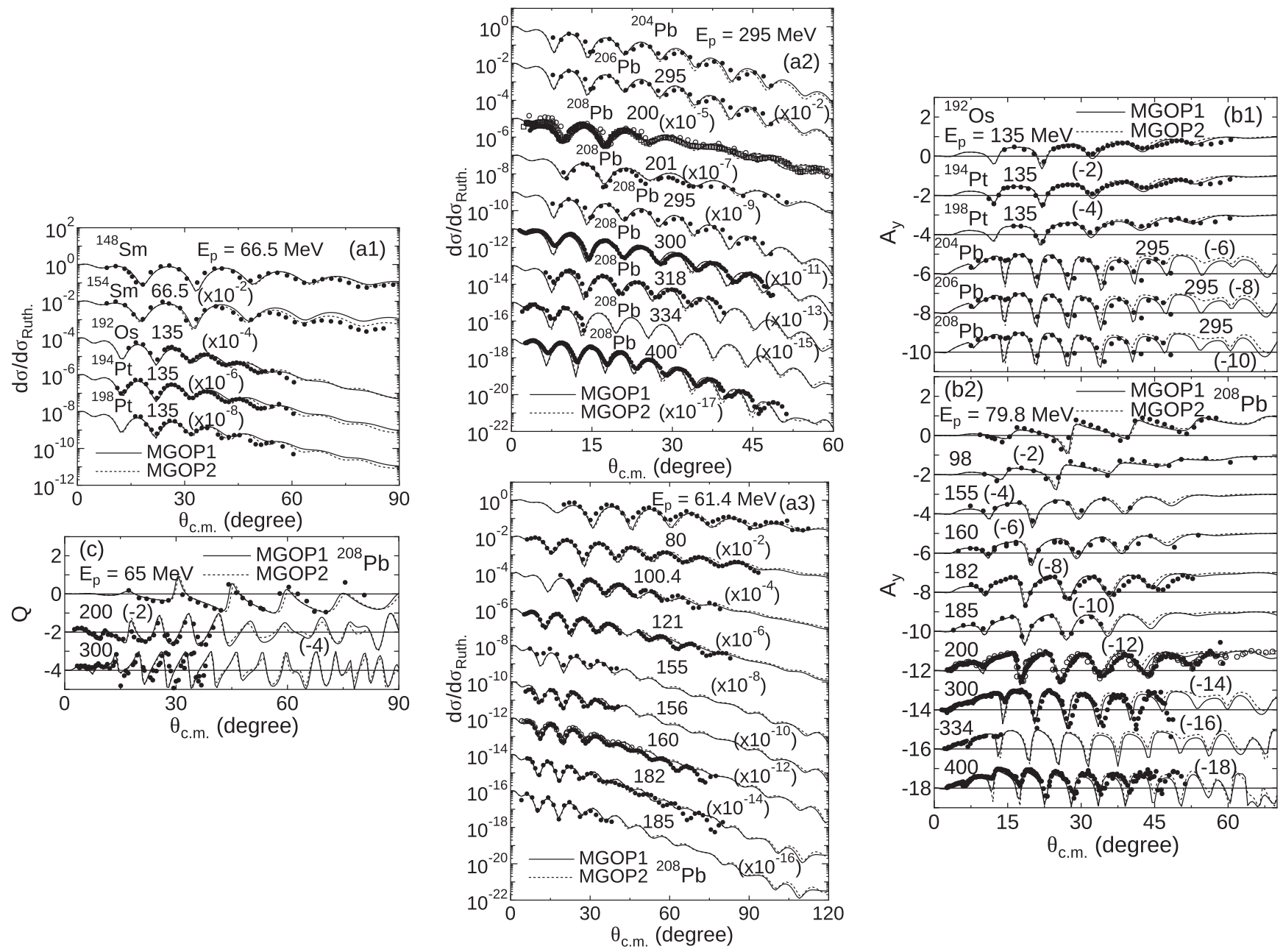

FIG. 25. (a) Elastic-scattering cross sections of $p+{ }^{148} \mathrm{Sm},{ }^{154} \mathrm{Sm},{ }^{192} \mathrm{Os},{ }^{194} \mathrm{Pt},{ }^{198} \mathrm{Pt},{ }^{204} \mathrm{~Pb},{ }^{206} \mathrm{~Pb}$, and ${ }^{208} \mathrm{~Pb}$ systems in the Rutherford ratio at $E_{p}=61.4-400 \mathrm{MeV}$; (b) analyzing power of $p+{ }^{192} \mathrm{Os},{ }^{194} \mathrm{Pt},{ }^{198} \mathrm{Pt},{ }^{204} \mathrm{~Pb},{ }^{206} \mathrm{~Pb}$, and ${ }^{208} \mathrm{~Pb} s y s t e m s$ at $E_{p}=$ 79.8-400 MeV; and (c) spin-rotation function $Q$ of $p+{ }^{208} \mathrm{~Pb}$ system at $E_{p}=65-300 \mathrm{MeV}$. The experimental data are taken from Refs. [4,32,33,74,133,135,159,168,172,178,183,188,196-205]. 


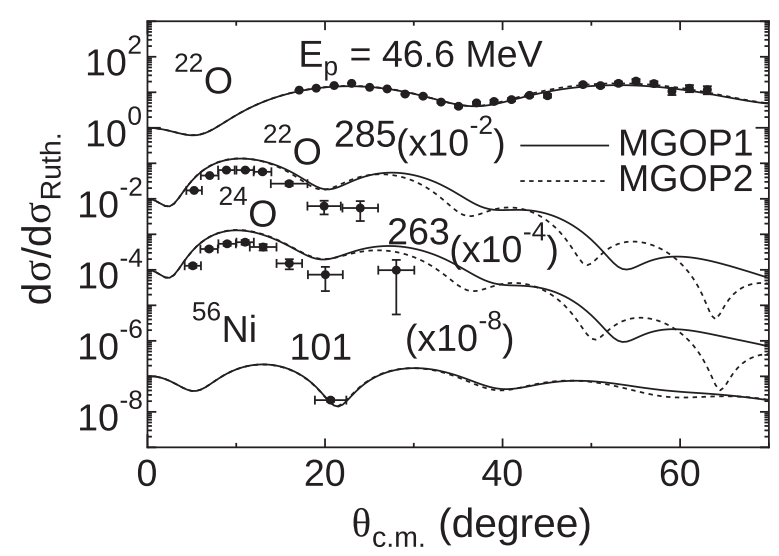

FIG. 26. Elastic-scattering cross sections in the Rutherford ratio of $p+{ }^{22} \mathrm{O},{ }^{24} \mathrm{O}$, and ${ }^{56} \mathrm{Ni}$ systems at $E_{p}=46.6-285 \mathrm{MeV}$. The experimental data are taken from Refs. [206-208].

complex $G$-matrix interaction. The SF model requires proton and neutron density distributions as inputs. We employ microscopic-mean-field models to obtain nuclear densities in a nonempirical way for a wide nuclear mass region including experimentally unknown nuclei. We examine two MGOP models constructed with input densities generated from the RMF and Skyrme-Hartree-Fock + BCS (HF + BCS) models.

By introducing the incident-energy dependent renormalization factor for the imaginary part, we find that both the

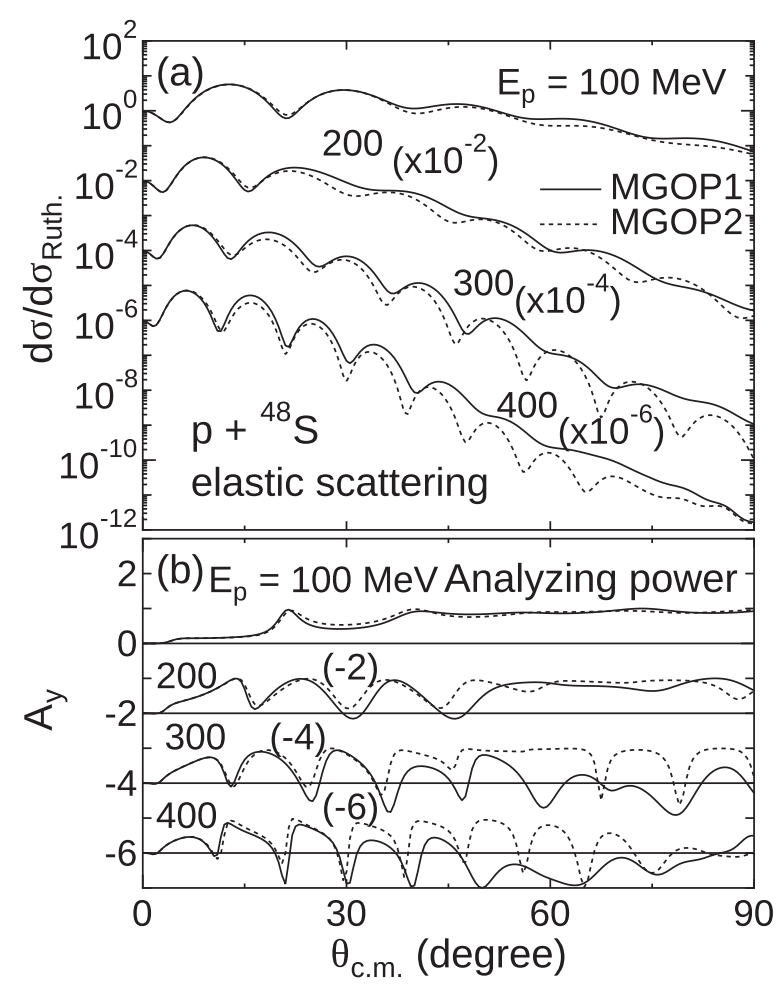

FIG. 27. (a) Elastic-scattering cross sections in the Rutherford ratio and (b) analyzing power of of $p+{ }^{48} \mathrm{~S}$ system at $E_{p}=$ 100-400 MeV. The MGOP1 (RMF) and MGOP2 (HF + BCS) models are employed.
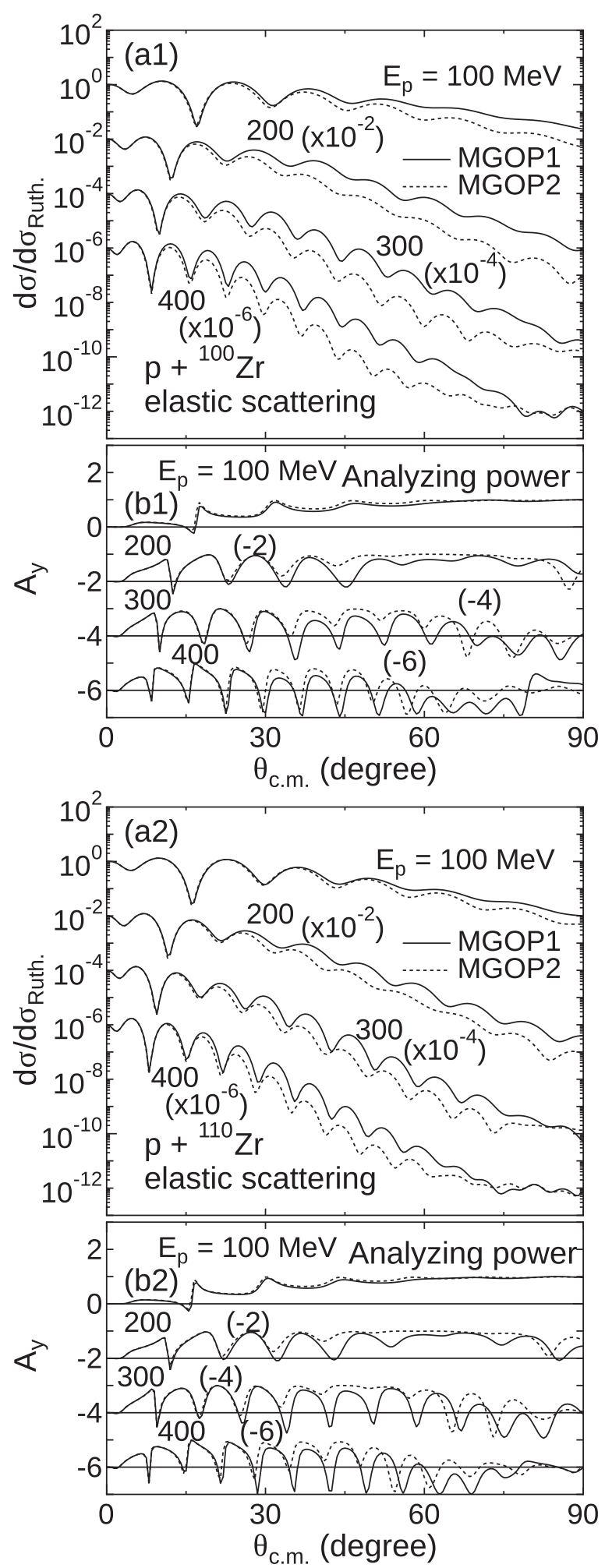

FIG. 28. (a) Elastic-scattering cross section in the Rutherford ratio and (b) analyzing power of $p+{ }^{100} \mathrm{Zr}$ and ${ }^{110} \mathrm{Zr}$ systems at $E_{p}=100-400 \mathrm{MeV}$. The MGOP1 (RMF) and MGOP2 (HF + BCS) models are employed.

present MGOP models well describe the nucleon-nucleus $(N A)$ scattering in a wide range of incident energies and nuclear mass numbers. We confirm that the validity of 
the present MGOPs in comparison to a number of the available experimental data of the $N A$ elastic-scattering cross sections, analyzing power, and spin-rotation function $Q$. The difference of the structure models is discussed for the proton scattering of ${ }^{48} \mathrm{~S}$ and neutron-rich $\mathrm{Zr}$ isotopes where the two mean-field models give quite different densities. We find that this difference in the cross sections becomes more apparent at the high incident energies $\gtrsim 200 \mathrm{MeV}$.

We provide the present MGOPs in a sum of the 12-range Gaussian functions whose parameters can be found together with a program source to reconstruct the MGOPs on the website [43]. We believe that the present MGOP models are useful to analyze various measurements as well as theoretical works involving unstable nuclear regions.

\section{ACKNOWLEDGMENTS}

We thank J. Zenihiro and D. Nishimura for the valuable comments. This work was partly supported by Japan Society for the Promotion of Science (JSPS) KAKENHI Grant Nos. JP15K17661, JP18K03635, and JP18H04569.
[1] G. R. Satchler, Direct Nuclear Reactions (Oxford University, Oxford, 1983).

[2] B. A. Watson, P. P. Singh, and R. E. Segel, Phys. Rev. 182, 977 (1969).

[3] J. F. D. Becchetti and G. W. Greenlees, Phys. Rev. 182, 1190 (1969).

[4] A. Nadasen, P. Schwandt, P. P. Singh, W. W. Jacobs, A. D. Bacher, P. T. Debevec, M. D. Kaitchuck, and J. T. Meek, Phys. Rev. C 23, 1023 (1981).

[5] S. Hama, B. C. Clark, E. D. Cooper, H. S. Sherif, and R. L. Mercer, Phys. Rev. C 41, 2737 (1990).

[6] R. L. Varner, W. J. Thompson, T. L. McAbee, E. J. Ludwig, and T. B. Clegg, Phys. Rep. 201, 57 (1991).

[7] E. D. Cooper, S. Hama, B. C. Clark, and R. L. Mercer, Phys. Rev. C 47, 297 (1993).

[8] A. J. Koning and J. P. Delaroche, Nucl. Phys. A 713, 231 (2003).

[9] Y. Han, Y. Xu, H. Liang, H. Guo, and Q. Shen, Phys. Rev. C 81, 024616 (2010).

[10] R. Xu, Z. Ma, Y. Zhang, Y. Tian, E. N. E. van Dalen, and H. Müther, Phys. Rev. C 94, 034606 (2016).

[11] C. M. Perey and F. G. Perey, Phys. Rev. 132, 755 (1963).

[12] W. W. Daehnick, J. D. Childs, and Z. Vrcelj, Phys. Rev. C 21, 2253 (1980).

[13] J. Bojowald, H. Machner, H. Nann, W. Oelert, M. Rogge, and P. Turek, Phys. Rev. C 38, 1153 (1988).

[14] H. An and C. Cai, Phys. Rev. C 73, 054605 (2006).

[15] Y. Han, Y. Shi, and Q. Shen, Phys. Rev. C 74, 044615 (2006).

[16] X. Li, C. Liang, and C. Cai, Nucl. Phys. A 789, 103 (2007).

[17] C.-T. Liang, X.-H. Li, and C.-H. Cai, J. Phys. G: Nucl. Part. Phys. 36, 085104 (2009).

[18] M. Nolte, H. Machner, and J. Bojowald, Phys. Rev. C 36, 1312 (1987).

[19] V. Avrigeanu, P. E. Hodgson, and M. Avrigeanu, Phys. Rev. C 49, 2136 (1994).

[20] A. Kumar, S. Kailas, S. Rathi, and K. Mahata, Nucl. Phys. A 776, 105 (2006).

[21] J. Cook, Nucl. Phys. A 388, 153 (1982).

[22] Y. Kucuk, I. Boztosun, and T. Topel, Phys. Rev. C 80, 054602 (2009).

[23] X. W. Su, Y. L. Han, H. Y. Liang, Z. D. Wu, H. R. Guo, and C. H. Cai, Phys. Rev. C 95, 054606 (2017).

[24] J. P. Jeukenne, A. Lejeune, and C. Mahaux, Phys. Rep. 25, 83 (1976).

[25] F. A. Brieva and J. R. Rook, Nucl. Phys. A 291, 299 (1977).
[26] J. P. Jeukenne, A. Lejeune, and C. Mahaux, Phys. Rev. C 16, 80 (1977).

[27] F. A. Brieva and J. R. Rook, Nucl. Phys. A 291, 317 (1977).

[28] F. A. Brieva and J. R. Rook, Nucl. Phys. A 297, 206 (1978).

[29] L. Rikus, K. Nakano, and H. V. V. Geramb, Nucl. Phys. A 414, 413 (1984).

[30] L. Rikus and H. V. V. Geramb, Nucl. Phys. A 426, 496 (1984).

[31] N. Yamaguchi, S. Nagata, and T. Matsuda, Prog. Theor. Phys. 70, 459 (1983).

[32] N. Yamaguchi, S. Nagata, and J. Michiyama, Prog. Theor. Phys. 76, 1289 (1986).

[33] H. F. Arellano, F. A. Brieva, and W. G. Love, Phys. Rev. C 52, 301 (1995).

[34] E. Bauge, J. P. Delaroche, and M. Girod, Phys. Rev. C 58, 1118 (1998).

[35] K. Amos, P. J. Dortmans, H. V. von Geramb, S. Karataglidis, and J. Raynal, Adv. Nucl. Phys. 25, 276 (2000).

[36] T. Furumoto, Y. Sakuragi, and Y. Yamamoto, Phys. Rev. C 78, 044610 (2008).

[37] K. Minomo, K. Ogata, M. Kohno, Y. R. Shimizu, and M. Yahiro, J. Phys. G: Nucl. Part. Phys. 37, 085011 (2010).

[38] K. Mizuyama and K. Ogata, Phys. Rev. C 86, 041603 (2012).

[39] M. Toyokawa, M. Yahiro, T. Matsumoto, K. Minomo, K. Ogata, and M. Kohno, Phys. Rev. C 92, 024618 (2015).

[40] G. Blanchon, M. Dupuis, H. F. Arellano, and N. VinhMau, Phys. Rev. C 91, 014612 (2015).

[41] L. C. Chamon, B. V. Carlson, L. R. Gasques, D. Pereira, C. DeConti, M. A. G. Alvarez, M. S. Hussein, M. A. Cândido Ribeiro, E. S. Rossi, Jr., and C. P. Silva, Phys. Rev. C 66, 014610 (2002).

[42] T. Furumoto, W. Horiuchi, M. Takashina, Y. Yamamoto, and Y. Sakuragi, Phys. Rev. C 85, 044607 (2012).

[43] http://www2.yukawa.kyoto-u.ac.jp/ takenori.furumoto/.

[44] T. Furumoto, Y. Sakuragi, and Y. Yamamoto, Phys. Rev. C 80, 044614 (2009).

[45] K. Tsubakihara and A. Ohnishi, Prog. Theor. Phys. 117, 903 (2007).

[46] K. Tsubakihara, H. Maekawa, H. Matsumiya, and A. Ohnishi, Phys. Rev. C 81, 065206 (2010).

[47] S. Ebata, T. Nakatsukasa, T. Inakura, K. Yoshida, Y. Hashimoto, and K. Yabana, Phys. Rev. C 82, 034306 (2010).

[48] Y. Yamamoto, T. Furumoto, N. Yasutake, and T. A. Rijken, Phys. Rev. C 88, 022801(R) (2013). 
[49] Y. Yamamoto, T. Furumoto, N. Yasutake, and T. A. Rijken, Phys. Rev. C 90, 045805 (2014).

[50] T. A. Rijken, M. M. Nagels, and Y. Yamamoto, Prog. Theor. Phys. Suppl. 185, 14 (2010).

[51] W. W. Qu et al., Phys. Lett. B 751, 1 (2015).

[52] T. Furumoto, Y. Sakuragi, and Y. Yamamoto, Phys. Rev. C 94, 044620 (2016)

[53] W. W. Qu et al., Phys. Rev. C 95, 044616 (2017).

[54] T. Furumoto, T. Suhara, and N. Itagaki, Phys. Rev. C 97, 044602 (2018).

[55] M. Isaka, Y. Yamamoto, and T. A. Rijken, Phys. Rev. C 94 044310 (2016).

[56] M. Isaka, Y. Yamamoto, and T. A. Rijken, Phys. Rev. C 95, 044308 (2017).

[57] Y. Yamamoto, T. Furumoto, N. Yasutake, and T. A. Rijken, Eur. Phys. J A 52, 19 (2016).

[58] J. W. Negele and D. M. Vautherin, Phys. Rev. C 5, 1472 (1972).

[59] X. Campi and A. Bouyssy, Phys. Lett. B 73, 263 (1978).

[60] D. T. Khoa, Phys. Rev. C 63, 034007 (2001).

[61] J. W. Negele, Phys. Rev. C 1, 1260 (1970).

[62] P. Ring and P. Schuck, The Nuclear Many-Body Problems (Springer-Verlag, New York, 1980).

[63] N. Tajima, S. Takahara, and N. Onishi, Nucl. Phys. A 603, 23 (1996).

[64] W. Horiuchi, T. Inakura, T. Nakatsukasa, and Y. Suzuki, Phys. Rev. C 86, 024614 (2012).

[65] J. Bartel, P. Quentin, M. Brack, C. Guet, and H. H. Kansson, Nucl. Phys. A 386, 79 (1982).

[66] W. Horiuchi, T. Inakura, T. Nakatsukasa, and Y. Suzuki, JPS Conf. Proc. 6, 030079 (2015).

[67] W. Horiuchi, Y. Suzuki, and T. Inakura, Phys. Rev. C 89, 011601(R) (2014).

[68] W. Horiuchi, S. Hatakeyama, S. Ebata, and Y. Suzuki, Phys. Rev. C 93, 044611 (2016).

[69] W. Horiuchi, S. Hatakeyama, S. Ebata, and Y. Suzuki, Phys. Rev. C 96, 024605 (2017).

[70] I. Angeli and K. Marinova, At. Data Nucl. Data Tables 99, 69 (2013).

[71] T. Janssens, R. Hofstadter, E. Hughes, and M. Ykarian, Phys. Rev. 142, 922 (1966).

[72] M. Grasso, L. Gaudefroy, E. Khan, T. Nikšić, J. Piekarewicz, O. Sorlin, N. VanGiai, and D. Vretenar, Phys. Rev. C 79, 034318 (2009).

[73] H. Nakada, K. Sugiura, and J. Margueron, Phys. Rev. C 87, 067305 (2013).

[74] http://www.jcprg.org/exfor/.

[75] J. M. Cassels and J. D. Lawson, Proc. Phys. Soc. (London) A 67, 125 (1954).

[76] E. J. Burge, Nucl. Phys. 13, 511 (1959).

[77] V. Meyer, R. M. Eisberg, and R. F. Carlson, Phys. Rev. 117, 1334 (1960).

[78] A. Johansson, U. Svanberg, and O. Sundberg, Arkiv Fysik 19, 527 (1961).

[79] R. Goloskie and K. Strauch, Nucl. Phys. 29, 474 (1962).

[80] P. Kirkby and W. T. Link, Can. J. Phys. 44, 1847 (1966).

[81] J. J. H. Menet, E. E. Gross, J. J. Malanify, and A. Zucker, Phys. Rev. Lett. 22, 1128 (1969).

[82] J. J. Menet, E. E. Gross, J. J. Malanify, and A. Zucker, Phys. Rev. C 4, 1114 (1971).
[83] P. U. Renberg, D. F. Measday, M. Pepin, P. Schwaller, B. Favier, and C. Richard-Serre, Nucl. Phys. A 183, 81 (1972).

[84] A. Ingemarsson et al., Nucl. Phys. A 653, 341 (1999).

[85] A. Auce et al., Phys. Rev. C 71, 064606 (2005).

[86] R. G. P. Voss and R. Wilson, Proc. R. Soc. London A 236, 41 (1956).

[87] W. Schimmerling, T. J. Devlin, W. W. Johnson, K. G. Vosburgh, and R. E. Mischke, Phys. Rev. C 7, 248 (1973).

[88] P. J. Dimbylow, Phys. Med. Bio. 25, 637 (1980).

[89] C. I. Zanelli, P. P. Urone, J. L. Romero, F. P. Brady, M. L. Johnson, G. A. Needham, J. L. Ullmann, and D. L. Johnson, Phys. Rev. C 23, 1015 (1981).

[90] M. Ibaraki, M. Baba, T. Miura, T. Aoki, T. Hiroishi, H. Nakashima, S. Meigo, and S. Tanaka, J. Nucl. Sci. Tech. Suppl. 39, 405 (2002).

[91] R. W. Finlay, W. P. Abfalterer, G. Fink, E. Montei, T. Adami, P. W. Lisowski, G. L. Morgan, and R. C. Haight, Phys. Rev. C 47, 237 (1993).

[92] W. P. Abfalterer, F. B. Bateman, F. S. Dietrich, R. W. Finlay, R. C. Haight, and G. L. Morgan, Phys. Rev. C 63, 044608 (2001).

[93] F. S. Dietrich et al., Phys. Rev. C 67, 044606 (2003).

[94] G. L. Salmon, Nucl. Phys. 21, 15 (1960).

[95] M. Ibaraki, M. Baba, T. Miura, Y. Hirasawa, Y. Nauchi, H. Nakashima, S.-I. Meigo, O. Iwamoto, and S. Tanaka, Nucl. Instrum. Methods Phys. Res. A 446, 536 (2000).

[96] J. Klug et al., Phys. Rev. C 68, 064605 (2003).

[97] J. H. Osborne et al., Phys. Rev. C 70, 054613 (2004).

[98] P. Mermod et al., Phys. Rev. C 74, 054002 (2006).

[99] A. Ohrn et al., Phys. Rev. C 77, 024605 (2008).

[100] I. C. Sagrado García, J. F. Lecolley, F. R. Lecolley, V. Blideanu, G. Ban, J. M. Fontbonne, G. Itis, J. L. Lecouey, T. Lefort, N. Marie, J. C. Steckmeyer, C. Le Brun, J. Blomgren, C. Johansson, J. Klug, A. Orhn, P. Mermod, N. Olsson, S. Pomp, M. Osterlund, U. Tippawan, A. V. Prokofiev, P. NadelTuronski, M. Fallot, Y. Foucher, A. Guertin, F. Haddad, and M. Vatre, Phys. Rev. C 84, 044619 (2011).

[101] A. Bratenahl, S. Fernbach, R. H. Hildebrand, C. E. Leith, and B. J. Moyer, Phys. Rev. 77, 597 (1950).

[102] C. P. V. Zyl, R. G. P. Voss, and R. Wilson, Philos. Mag. 1, 1003 (1956).

[103] A. Ashmore, D. S. Mather, and S. K. Sen, Proc. Phys. Soc. (London) 71, 552 (1958).

[104] E. L. Hjort, F. P. Brady, J. L. Romero, J. R. Drummond, D. S. Sorenson, J. H. Osborne, B. McEachern, and L. F. Hansen, Phys. Rev. C 50, 275 (1994).

[105] P. H. Bowen, G. C. Cox, G. B. Huxtable, J. P. Scanlon, J. J. Thresher, A. Langsford, and H. Appel, Nucl. Phys. 40, 186 (1963).

[106] H. Sakaguchi, M. Nakamura, K. Hatanaka, A. Goto, T. Noro, F. Ohtani, H. Sakamoto, and S. Kobayashi, Phys. Lett. B 89, 40 (1979).

[107] S. Kato et al., Nucl. Instrum. Methods 169, 589 (1980).

[108] H. Ejiri, M. Sasao, T. Shibata, H. Ohsumi, Y. Fujita, M. Fujiwara, T. Yamazaki, I. Katayama, S. Morinobu, and H. Ikegami, Phys. Rev. C 24, 2001 (1981).

[109] T. Noro, H. Sakaguchi, M. Nakamura, K. Hatanaka, F. Ohtani, H. Sakamoto, and S. Kobayashi, Nucl. Phys. A 366, 189 (1981). 
[110] H. Sakaguchi, M. Nakamura, K. Hatanaka, T. Noro, F. Ohtani, H. Sakamoto, H. Ogawa, and S. Kobayashi, Phys. Lett. B 99, 92 (1981).

[111] H. Sakaguchi, M. Nakamura, K. Hatanaka, A. Goto, T. Noro, F. Ohtani, H. Sakamoto, H. Ogawa, and S. Kobayashi, Phys. Rev. C 26, 944 (1982).

[112] M. Nakamura, H. Sakaguchi, H. Sakamoto, H. Ogawa, O. Cynshi, S. Kobayashi, S. Kato, N. Matsuoka, K. Hatanaka, and T. Noro, Nucl. Instrum. Methods Phys. Res. 212, 173 (1983).

[113] F. Ohtani, H. Sakaguchi, M. Nakamura, T. Noro, H. Sakamoto, H. Ogawa, T. Ichihara, M. Yosoi, and S. Kobayashi, Phys. Rev. C 28, 120 (1983).

[114] T. Ichihara et al., Phys. Rev. C 29, 1228 (1984).

[115] S. Kato et al., Phys. Rev. C 31, 1616 (1985).

[116] H. Ogawa et al., Phys. Rev. C 33, 834 (1986).

[117] Y. Takeuchi, H. Sakaguchi, M. Nakamura, T. Ichihara, M. Yosoi, M. Ieiri, and S. Kobayashi, Phys. Rev. C 34, 493 (1986).

[118] T. Ichihara, H. Sakaguchi, M. Nakamura, M. Yosoi, M. Ieiri, Y. Takeuchi, H. Togawa, T. Tsutsumi, and S. Kobayashi, Phys. Rev. C 36, 1754 (1987).

[119] M. Fujiwara et al., Phys. Rev. C 35, 1257 (1987).

[120] R. DeLeo et al., Phys. Rev. C 53, 2718 (1996).

[121] R. E. Richardson, W. P. Ball, C. E. Leith, and B. J. Moyer, Phys. Rev. 86, 29 (1952).

[122] K. Strauch and F. Titus, Phys. Rev. 103, 200 (1956).

[123] R. Alphonce, A. Johansson, and G. Tibell, Nucl. Phys. 3, 185 (1957).

[124] R. Alphonce, A. Johansson, and G. Tibell, Nucl. Phys. 4, 672 (1957).

[125] P. Hillman, A. Johansson, and H. Tyren, Nucl. Phys. 4, 648 (1957).

[126] J. -P. Garron, J. -C. Jacmart, L. Massonnet, M. Riou, and C. Ruhla, J. Phys. 21, 317 (1960).

[127] A. Johansson, U. Svanberg, and P. E. Hodgson, Arkiv Fysik 19, 541 (1961).

[128] A. E. Taylor and E. Wood, Nucl. Phys. 25, 642 (1961).

[129] I. Nonaka, Y. Saji, A. Suzuki, H. Yamaguchi, R. Eisberg, Y. Ishisaki, K. Kikuchi, K. Matsuda, T. Mikumo, and Y. Nakajima, J. Phys. Soc. Jpn. 17, 1817 (1962).

[130] D. J. Steinberg, J. N. Palmieri, and A. M. Cormack, Nucl. Phys. 56, 46 (1964).

[131] J. M. Emmerson, J. C. W. Madden, C. M. P.Johnson, N. Middlemas, A. B. Clegg, and W. S. C. Williams, Nucl. Phys. 77, 305 (1966).

[132] C. Rolland, B. Geoffrion, N. Marty, M. Morlet, B. Tatischeff, and A. Willis, Nucl. Phys. 80, 625 (1966).

[133] C. B. Fulmer, J. B. Ball, A. Scott, and M. L. Whiten, Phys. Rev. 181, 1565 (1969).

[134] F. E. Bertrand and R. W. Peelle, Phys. Rev. C 8, 1045 (1973).

[135] V. Comparat, R. Frascaria, N. Marty, M. Morlet, and A. Willis, Nucl. Phys. A 221, 403 (1974).

[136] A. Ingemarsson, O. Jonsson, and A. Hallgren, Nucl. Phys. A 319, 377 (1979).

[137] H. Ohnuma, J. Kasagi, F. Kakimoto, S. Kubono, and K. Koyama, J. Phys. Soc. Jpn. 48, 1812 (1980).

[138] J. R. Comfort, S. M. Austin, P. T. Debevec, G. L. Moake, R. W. Finlay, and W. G. Love, Phys. Rev. C 21, 2147 (1980).

[139] H. O. Meyer, P. Schwandt, G. L. Moake, and P. P. Singh, Phys. Rev. C 23, 616 (1981).

[140] W. Bauhoff et al., Nucl. Phys. A 410, 180 (1983).
[141] H. O. Meyer, P. Schwandt, W. W. Jacobs, and J. R. Hall, Phys. Rev. C 27, 459 (1983).

[142] D. Ottewell, P. Walden, E. G. Auld, G. Giles, G. Jones, G. J. Lolos, B. J. McParland, W. Ziegler, and W. Falk, Nucl. Phys. 412, 189 (1984).

[143] H. O. Meyer et al., Phys. Rev. C 31, 1569 (1985).

[144] M. Ieiri et al., Nucl. Instrum. Methods A 257, 253 (1987).

[145] H. O. Meyer et al., Phys. Rev. C 37, 544 (1988).

[146] S. W. Wissink et al., Phys. Rev. C 45, R504 (1992).

[147] Z. Yu, R. E. Segel, T.-Y. Tung, R. D. Bent, C. C. Foster, J. Goodwin, G. Hardie, and J. Homolka, Phys. Rev. C 53, 1725 (1996).

[148] V. M. Hannen et al., Phys. Rev. C 67, 054320 (2003).

[149] A. Okamoto et al., Phys. Rev. C 81, 054604 (2010).

[150] K. Amos et al., Nucl. Phys. A 413, 255 (1984).

[151] C. W. Glover, P. Schwandt, H. O. Meyer, W. W. Jacobs, J. R. Hall, M. D. Kaitchuck, and R. P. DeVito, Phys. Rev. C 31, 1 (1985).

[152] J. J. Kelly et al., Phys. Rev. C 39, 1222 (1989).

[153] J. J. Kelly et al., Phys. Rev. C 41, 2504 (1990).

[154] J. J. Kelly et al., Phys. Rev. C 43, 1272 (1991).

[155] H. Seifert et al., Phys. Rev. C 47, 1615 (1993).

[156] T. Wakasa et al., Phys. Lett. B 632, 485 (2006).

[157] O. Sundberg, A. Johansson, G. Tibell, S. Dahlgren, D. Hasselgren, B. Hoistad, A. Ingemarsson, and P.-U. Renberg, Nucl. Phys. A 101, 481 (1967).

[158] B. Hoistad, A. Ingemarsson, A. Johansson, and G. Tibell, Nucl. Phys. A 119, 290 (1968).

[159] P. Schwandt, H. O. Meyer, W. W. Jacobs, A. D. Bacher, S. E. Vigdor, M. D. Kaitchuck, and T. R. Donoghue, Phys. Rev. C 26, 55 (1982).

[160] C. Olmer, A. D. Bacher, G. T. Emery, W. P. Jones, D. W. Miller, H. Nann, P. Schwandt, S. Yen, T. E. Drake, and R. J. Sobie, Phys. Rev. C 29, 361 (1984).

[161] K. Hatanaka, M. Fujiwara, K. Hosono, N. Matsuoka, T. Saito, and H. Sakai, Phys. Rev. C 29, 13 (1984).

[162] K. H. Hicks et al., Phys. Rev. C 38, 229 (1988).

[163] Q. Chen, J. J. Kelly, P. P. Singh, M. C. Radhakrishna, W. P. Jones, and H. Nann, Phys. Rev. C 41, 2514 (1990).

[164] J. J. Kelly, Q. Chen, P. P. Singh, M. C. Radhakrishna, W. P. Jones, and H. Nann, Phys. Rev. C 41, 2525 (1990).

[165] J. J. Kelly et al., Phys. Rev. C 44, 1963 (1991).

[166] J. J. Kelly et al., Phys. Rev. C 44, 2602 (1991).

[167] K. Yagi et al., Phys. Lett. 10, 186 (1964).

[168] P. G. Roos and N. S. Wall, Phys. Rev. 140, B1237 (1965).

[169] J. Kallne, G. Tibell, and A. Johansson, Phys. Scr. 2, 195 (1970).

[170] D. J. Horen et al., Phys. Rev. C 31, 2049 (1985).

[171] D. Frekers et al., Phys. Rev. C 35, 2236 (1987).

[172] D. A. Hutcheon et al., Nucl. Phys. A 483, 429 (1988).

[173] A. E. Feldman et al., Phys. Rev. C 49, 2068 (1994).

[174] A. Johansson, G. Tibell, and P. Hillman, Nucl. Phys. 11, 540 (1959).

[175] M. Koike, K. Matsuda, I. Nonaka, Y. Saji, K. Yagi, H. Ejiri, Y. Ishizaki, Y. Nakajima, and E. Tanaka, J. Phys. Soc. Jpn. 21, 2103 (1966).

[176] K. Yagi, Y. Saji, Y. Ishizaki, T. Ishimatsu, Y. Nakajima, M. Matoba, and C. Y. Huang, Nucl. Phys. A 132, 690 (1969).

[177] D. C. Kocher, F. E. Bertrand, E. E. Gross, and E. Newman, Phys. Rev. C 14, 1392 (1976). 
[178] K. Kwiatkowski and N. S. Wall, Nucl. Phys. A 301, 349 (1978).

[179] A. Ingemarsson, T. Johansson, and G. Tibell, Nucl. Phys. A 322, 285 (1979).

[180] A. Ingemarsson, T. Johansson, and G. Tibell, Nucl. Phys. A 365, 426 (1981).

[181] L. W. Woo, N. S. Wall, P. G. Roos, P. H. Debenham, K. Kwiatkowski, and A. Nadasen, Phys. Rev. C 29, 794 (1984).

[182] K. Ogino, Phys. Rev. C 33, 71 (1986).

[183] N. M. Hintz et al., Phys. Rev. C 37, 692 (1988).

[184] O. Hausser et al., Phys. Rev. C 43, 230 (1991).

[185] H. Sakaguchi et al., Phys. Rev. C 57, 1749 (1998).

[186] H. Takeda, Memoirs Faculty of Sci., Kyoto Univ., Ser. Phys. 44, 1 (2003).

[187] F. Hofmann et al., Phys. Lett. B 612, 165 (2005).

[188] J. Zenihiro et al., Phys. Rev. C 82, 044611 (2010).

[189] E. Hagberg, A. Ingemarsson, and B. Sundqvist, Phys. Scr. 3, 245 (1971).

[190] P. Martin, Y. Gaillard, P. de Saintignon, G. Perrin, J. Chauvin, G. Duhamel, and J. M. Loiseaux, Nucl. Phys. A 315, 291 (1979).

[191] S. Kailas, P. P. Singh, D. L. Friesel, C. C. Foster, P. Schwandt, and J. Wiggins, Phys. Rev. C 29, 2075 (1984).

[192] J. J. Kelly et al., Phys. Rev. C 47, 2146 (1993).

[193] M. Koike, T. Suehiro, K. Pingel, K. Komura, I. Nonaka, T. Wada, T. Fujisawa, H. Kamitsubo, and T. Nojiri, Nucl. Phys. A 248, 237 (1975).

[194] M. Pignanelli, S. Micheletti, E. Cereda, M. N. Harakeh, S. Y. vanderWerf, and R. DeLeo, Phys. Rev. C 29, 434 (1984).
[195] S. Terashima et al., Phys. Rev. C 77, 024317 (2008).

[196] A. Willis, B. Geoffrion, N. Marty, M. Morlet, C. Rolland, and B. Tatischeff, Nucl. Phys. A 112, 417 (1968).

[197] W. T. H. van Oers, H. Haw, N. E. Davison, A. Ingemarsson, B. Fagerstrom, and G. Tibell, Phys. Rev. C 10, 307 (1974).

[198] C. Djalali, N. Marty, M. Morlet, and A. Willis, Nucl. Phys. A 380, 42 (1982).

[199] F. E. Bertrand et al., Phys. Rev. C 34, 45 (1986).

[200] D. K. McDaniels et al., Phys. Rev. C 33, 1943 (1986).

[201] D. Cook, N. M. Hintz, M. M. Gazzaly, G. Pauletta, R. W. Fergerson, G. W. Hoffmann, J. B. McClelland, and K. W. Jones, Phys. Rev. C 35, 456 (1987).

[202] L. Lee et al., Phys. Lett. B 205, 219 (1988).

[203] F. T. Baker, A. Sethi, V. Penumetcha, G. T. Emery, W. P. Jones, M. A. Grimm, and M. L. Whiten, Nucl. Phys. A 501, 546 (1989).

[204] A. Guterman, D. L. Hendrie, P. H. Debenham, K. Kwiatkowski, A. Nadasen, L. W. Woo, and R. M. Ronningen, Phys. Rev. C 39, 1730 (1989).

[205] A. Sethi, F. T. Baker, G. T. Emery, W. P. Jones, and J. M. A. Grimm, Nucl. Phys. A 518, 536 (1990).

[206] G. Kraus et al., Phys. Rev. Lett. 73, 1773 (1994).

[207] E. Becheva et al., Phys. Rev. Lett. 96, 012501 (2006).

[208] S. Boissinot, Ph.D. Thesis, University Paris-Sud XI (2013), https://tel.archives-ouvertes.fr/tel-00958441.

[209] S. Hatakeyama, W. Horiuchi, and A. Kohama, Phys. Rev. C 97, 054607 (2018).

[210] E. Cheifetz, R. C. Jared, S. G. Thompson, and J. B. Wilhelmy, Phys. Rev. Lett. 25, 38 (1970).

[211] P. Sarriguren and J. Pereira, Phys. Rev. C 81, 064314 (2010).

[212] N. Paul et al., Phys. Rev. Lett. 118, 032501 (2017). 